\title{
IntelliO3-ts v1.0: a neural network approach to predict near-surface ozone concentrations in Germany
}

\author{
Felix Kleinert ${ }^{1,2}$, Lukas H. Leufen ${ }^{1,2}$, and Martin G. Schultz ${ }^{1}$ \\ ${ }^{1}$ Jülich Supercomputing Centre, Forschungszentrum Jülich, Jülich, Germany \\ ${ }^{2}$ Institute of Geosciences, Rheinische Friedrich-Wilhelms-Universität Bonn, Bonn, Germany \\ Correspondence: Felix Kleinert (f.kleinert@fz-juelich.de)
}

Received: 3 June 2020 - Discussion started: 17 August 2020

Revised: 6 November 2020 - Accepted: 7 November 2020 - Published: 4 January 2021

\begin{abstract}
The prediction of near-surface ozone concentrations is important for supporting regulatory procedures for the protection of humans from high exposure to air pollution. In this study, we introduce a data-driven forecasting model named "IntelliO3-ts", which consists of multiple convolutional neural network (CNN) layers, grouped together as inception blocks. The model is trained with measured multi-year ozone and nitrogen oxide concentrations of more than 300 German measurement stations in rural environments and six meteorological variables from the meteorological COSMO reanalysis. This is by far the most extensive dataset used for time series predictions based on neural networks so far. IntelliO3-ts allows the prediction of daily maximum $8 \mathrm{~h}$ average (dma8eu) ozone concentrations for a lead time of up to $4 \mathrm{~d}$, and we show that the model outperforms standard reference models like persistence models. Moreover, we demonstrate that IntelliO3-ts outperforms climatological reference models for the first $2 \mathrm{~d}$, while it does not add any genuine value for longer lead times. We attribute this to the limited deterministic information that is contained in the single-station time series training data. We applied a bootstrapping technique to analyse the influence of different input variables and found that the previous-day ozone concentrations are of major importance, followed by $2 \mathrm{~m}$ temperature. As we did not use any geographic information to train IntelliO3-ts in its current version and included no relation between stations, the influence of the horizontal wind components on the model performance is minimal. We expect that the inclusion of advection-diffusion terms in the model could improve results in future versions of our model.
\end{abstract}

\section{Introduction}

Exposure to ambient air pollutants such as ozone $\left(\mathrm{O}_{3}\right)$ is harmful for living beings (WHO, 2013; Bell et al., 2014; Lefohn et al., 2017; Fleming et al., 2018) and certain crops (Avnery et al., 2011; Mills et al., 2018). Therefore, the prediction of ozone concentrations is of major importance for issuing warnings for the public if high ozone concentrations are foreseeable. As tropospheric ozone is a secondary air pollutant, there is nearly no source of directly emitted ozone. Instead, it is formed in chemical reactions of several precursors like nitrogen oxides $\left(\mathrm{NO}_{x}\right)$ or volatile organic compounds (VOCs). Weather conditions (temperature, irradiation, humidity, and winds) have a major influence on the rates of ozone formation and destruction. Ozone has a "chemical lifetime" in the lower atmosphere of several days and can therefore be transported over distances of several hundred kilometres.

Ozone concentrations can be forecasted by various numerical methods. Chemical transport models (CTMs) solve chemical and physical equations explicitly (for example, Collins et al., 1997; Wang et al., 1998a, b; Horowitz et al., 2003; von Kuhlmann et al., 2003; Grell et al., 2005; Donner et al., 2011). These numerical models predict concentrations for grid cells, which are assumed to be representative of a given area. Therefore, local fluctuations which are below model resolution cannot be simulated. Moreover, CTMs often have a bias in concentrations, turnover rates, or meteorological properties which have a direct influence on chemical processes (Vautard, 2012; Brunner et al., 2015).

This makes CTMs unsuited for regulatory purposes, which by law are bound to station measurements, except if so-called 
model output statistics are applied to the numerical modelling results (Fuentes and Raftery, 2005). As an alternative to CTMs, regression models are often used to generate point forecasts (for example, Olszyna et al., 1997; Thompson et al., 2001; Abdul-Wahab et al., 2005). Regression models are pure statistical models, which are based on empirical relations among different variables. They usually describe a linear functional relationship between various factors (precursor concentrations, meteorological, and site information) and the air pollutant in question.

Since the late 1990s, machine learning techniques in the form of neural networks have also been applied as a regression technique to forecast ozone concentrations or threshold value exceedances (see Table 1). As the computational power was limited in the early days of those approaches, many of these early studies focused on a small number of measurement stations and used a fully connected (FC) network architecture. More recent studies explored the use of more advanced network architectures like convolutional neural networks (CNNs) or long short-term memory (LSTM) networks. These networks were also applied to a larger number of stations compared to the earlier studies and some studies have tried to generalise, i.e. to train one neural network for all stations instead of training individual networks for individual stations (Table 1). Although the total number of studies focusing on air quality or explicit near-surface ozone is already quite substantial, there are only few studies which applied advanced deep learning approaches on a larger number of stations or on longer time series. Eslami et al. (2020) applied a CNN on time series of 25 measurement stations in Seoul, South Korea, to predict hourly ozone concentrations for the next $24 \mathrm{~h}$. Ma et al. (2020) trained a bidirectional LSTM on 19 measurement sites over a period of roughly 9 months and afterwards used that model to re-train individually for 48 previously unused measurement stations (transfer learning).

Sayeed et al. (2020) applied a deep CNN on data from 21 different measurement stations over a period of 4 years. They used 3 years (2014 to 2016) to train their model and evaluated the generalisation capability on the fourth year (2017). Zhang et al. (2020) developed a hybrid CNN-LSTM model to predict gridded air quality concentrations $\left(\mathrm{O}_{3}, \mathrm{NO}_{2}, \mathrm{CO}\right.$, $\mathrm{PM}_{2.5}, \mathrm{PM}_{10}$ ). Seltzer et al. (2020) used 3557 measurement sites across six measurement networks to analyse long-term ozone exposure trends in North America by applying a fully connected neural network. They mainly focused on metrics related to human health and crop loss.

The current study extends these previous works and introduces a new deep learning model for the prediction of daily maximum $8 \mathrm{~h}$ average $\mathrm{O}_{3}$ concentrations (dma8eu; see Sect. 2.1) for a lead time of up to $4 \mathrm{~d}$. The network architecture is based on several stacks of convolutional neural networks. We trained our network with data from 312 background measurement stations in Germany (date range from 1997 to 2007) and tuned hyperparameters on data from 211 stations (data range from 2008 to 2009). We evaluate the performance at 203 stations, which have data during the 20102015 period looking at skill scores, the joint distribution of forecasts and observations, as well as the influence of input variables.

This article is structured as follows: in Sect. 2, we explain our variable selection and present our prepossessing steps. In Sect. 3, we introduce our forecasting model (IntelliO3ts, version 1.0). Section 4 introduces the statistical tools and reference models which were used for verification. In Sect. 5, we present and discuss the results and analyse the influence of different input variables on the model performance. In Sect. 6, we discuss the limitations of IntelliO3-ts. Finally, Sect. 7 provides conclusions.

\section{Variable selection and data processing}

\subsection{Variable selection}

Tropospheric ozone $\left(\mathrm{O}_{3}\right)$ is a greenhouse gas formed in the atmosphere by chemical reactions of other directly emitted pollutants (ozone precursors) and therefore referred to as a secondary air pollutant.

The continuity equation of near-surface ozone in a specific volume of air can be written as (Jacobson, 2005, p. 74ff)

$\frac{\partial N_{q}}{\partial t}+\nabla \cdot\left(\boldsymbol{v} N_{q}\right)=\left(\nabla \cdot \mathbf{K}_{h} \boldsymbol{\nabla}\right) N_{q}+R_{\text {depg }}+R_{\text {chemg }}$,

where $\frac{\partial N_{q}}{\partial t}$ is the partial derivative of the ozone number concentration with respect to time, $v$ is the vector wind velocity, $\mathbf{K}_{h}$ is the eddy diffusion tensor for energy, while $R_{\text {depg }}$ and $R_{\text {chemg }}$ are the rates of dry deposition to the ground, and photochemical production or loss, respectively.

Tropospheric ozone is formed under sunlit conditions in gas-phase chemical reactions of peroxy radicals and nitrogen oxides (Seinfeld and Pandis, 2016). The peroxy radicals are themselves oxidation products of volatile organic compounds. The nitrogen oxides undergo a rapid catalytic cycle:

$\mathrm{NO}_{2}+h v+\mathrm{O}_{2}+M \rightarrow \mathrm{NO}+\mathrm{O}_{3}+M^{*}$

$\mathrm{NO}+\mathrm{O}_{3} \rightarrow \mathrm{NO}_{2}+\mathrm{O}_{2}$,

where $\mathrm{NO}$ and $\mathrm{O}_{3}$ are converted to $\mathrm{NO}_{2}$ and back within minutes ( $\mathrm{M}$ is an arbitrary molecule which is needed to take up excess energy, denoted by the asterisk). As a consequence, ozone concentrations in urban areas with high levels of $\mathrm{NO}_{x}$ from combustive emissions are extremely variable. In this study, we therefore focus on background stations, which are less affected by the rapid chemical interconversion.

From a chemical perspective, the prediction of ozone concentrations would require concentration data of $\mathrm{NO}, \mathrm{NO}_{2}$, speciated VOC, and $\mathrm{O}_{3}$ itself. However, since speciated VOC measurements are only available from very few measurement sites, the chemical input variables of our model are limited to $\mathrm{NO}, \mathrm{NO}_{2}$, and $\mathrm{O}_{3}$. 
Table 1. Overview of the literature on ozone forecasts with neural networks. Machine learning (ML) types are abbreviated as FC for fully connected, CNN for convolutional neural network, RNN for recurrent neural network, and LSTM for long short-term memory. We use the following abbreviations for time periods: yr for years and $\mathrm{m}$ for month.

\begin{tabular}{|c|c|c|c|c|c|}
\hline Citation & ML type & $\begin{array}{l}\text { Total number of } \\
\text { stations }\end{array}$ & $\begin{array}{l}\text { Stations for } \\
\text { training }\end{array}$ & Time period & Comments \\
\hline Comrie (1997) & FC & 8 & 8 & $5 \mathrm{yr}$ & Random split for train, val \\
\hline Cobourn et al. (2000) & $\mathrm{FC}$ & 7 & 7 & $\begin{array}{l}5 \mathrm{yr}(\operatorname{train})+1 \mathrm{yr}(\mathrm{val})+1 \mathrm{yr} \\
(\text { test })\end{array}$ & \\
\hline Prybutok et al. (2000) & $\mathrm{FC}$ & 1 & & $4 m+1 m$ & \\
\hline Gardner and Dorling (2001) & $\mathrm{FC}$ & 6 & 6 & $12 \mathrm{yr}$ & \\
\hline Eslami et al. (2020) & $\mathrm{CNN}$ & 25 & 25 & $3 \mathrm{yr}$ train $1 \mathrm{yr}$ test & Random split \\
\hline Liu et al. (2019) & Attention RNN & 2 & 2 & $10 \mathrm{~m}$ (train), $7 \mathrm{~d}$ (test) & Analysis for $\mathrm{PM}_{2.5}$ \\
\hline Maleki et al. (2019) & $\mathrm{FC}$ & 4 & 4 & $1 \mathrm{yr}$ & Random split for train, val, test \\
\hline Silva et al. (2019) & $\mathrm{FC}$ & 2 & 1 & $\begin{array}{l}13 \mathrm{yr} \text { (train, val test); } 14 \mathrm{yr} \text { (test } \\
\text { on second station) }\end{array}$ & $\begin{array}{l}\text { Dry deposition of } \mathrm{O}_{3} \text {; random } \\
\text { split on first station }\end{array}$ \\
\hline Abdul Aziz et al. (2019) & FC & 1 & 1 & $7 d$ & $\begin{array}{l}\text { Individual measurements for } \\
\text { study }\end{array}$ \\
\hline Pawlak and Jarosławski (2019) & $\mathrm{FC}$ & 2 & 2 & $6 \mathrm{~m}$ (train) $+6 \mathrm{~m}$ (test) & Individual network per station \\
\hline Seltzer et al. (2020) & $\mathrm{FC}$ & 3557 & 3557 & $15 \mathrm{yr}$ & focus on trends \\
\hline Ma et al. (2020) & Bidir. LSTM & $\begin{array}{l}19 \text { (standard) }+ \\
48 \text { (transfer) }\end{array}$ & $\begin{array}{l}19 \text { (standard) }+ \\
48 \text { (transfer) }\end{array}$ & $9 \mathrm{~m}$ & Exploration of transfer learning \\
\hline Sayeed et al. (2020) & $\mathrm{CNN}$ & 21 & 21 & $3 \mathrm{yr}$ (train) $+1 \mathrm{yr}$ (test/re-train) & $\begin{array}{l}\text { Re-train model for each } \\
\text { prediction }\end{array}$ \\
\hline Zhang et al. (2020) & CNN LSTM & 35 & 35 & $19 \mathrm{~m}$ & Gridded forecast \\
\hline This study & Inception blocks & 329 & 312 & $18 \mathrm{yr}$ & \\
\hline
\end{tabular}

Besides the trace gas concentrations, ozone levels also depend on meteorological variables. Due to the scarcity of reported meteorological measurements at the air quality monitoring sites, we extracted time series of meteorological variables from the $6 \mathrm{~km}$ resolution COSMO reanalysis (Bollmeyer et al., 2015, COSMO-REA6) and treat those as observations.

All data used in this study were retrieved from the Tropospheric Ozone Assessment Report (TOAR) database (Schultz et al., 2017) via the representational state transfer (REST) application programming interface (API) at https: //join.fz-juelich.de (last access: 12 November 2020). The air quality measurements were provided by the German Umweltbundesamt, while the meteorological data were extracted from the COSMO-REA6 reanalysis as described above. These reanalysis data cover the period from 1995 to 2015 with some gaps due to incompleteness in the TOAR database. As discussed in the US EPA guidelines on air quality forecasting (Dye, 2003), ozone concentrations typically depend on temperature, irradiation, humidity, wind speed, and wind direction. The vertical structure of the lowest portion of the atmosphere (i.e. the planetary boundary layer) also plays an important role, because it determines the rate of mixing between fresh pollution and background air. Since irradiation data were not available from the REST interface, we used cloud cover together with temperature as proxy variables.

Table 2 shows the list of input variables used in this study, and Table 3 describes the daily statistics that were applied to the hourly data of each variable. The choice of using the dma8eu metrics for $\mathrm{NO}$ and $\mathrm{NO}_{2}$ was motivated by the idea
Table 2. Input variables and applied daily statistics according to Table 3.

\begin{tabular}{ll}
\hline Variable & Daily statistics \\
\hline $\mathrm{NO}$ & dma8eu \\
$\mathrm{NO}_{2}$ & dma8eu \\
$\mathrm{O}_{3}$ & dma8eu \\
Cloud cover & average \\
Planetary boundary layer height & maximum \\
Relative humidity & average \\
Temperature & maximum \\
Wind's $u$ component & average \\
Wind's $v$ component & average \\
\hline
\end{tabular}

to sample all chemical quantities during the same time periods and with similar averaging times. While the dma8eu metrics is calculated based on data starting at 17:00 LT on the previous day, the daily mean/max values, for example, would be calculated based on data starting from the current day. To test the impact of using different metrics for ozone precursors, we also trained the model from scratch with either mean or maximum concentrations of $\mathrm{NO}$ and $\mathrm{NO}_{2}$ as inputs. The results of these runs were hardly distinguishable from the results presented below.

As described above, ozone concentrations are less variable at stations, which are further away from primary pollutant emission sources. We therefore selected those stations from the German air quality monitoring network, which are labelled as "background" stations according to the European Environmental Agency (EEA), Airbase classification. 
Table 3. Definitions of statistical metrics in TOAR analysis relevant for this study. Adopted form Schultz et al. (2017, Supplement 1, Table 6, therein).

\begin{tabular}{ll}
\hline Name & Description \\
\hline $\begin{array}{l}\text { data_capture } \\
\text { average_values }\end{array}$ & $\begin{array}{l}\text { Fraction of valid (hourly) values available in the aggregation period. } \\
\text { Daily [...] average value. No data capture criterion is applied, i.e. a daily average is valid if at least one hourly } \\
\text { value of the day is present. }\end{array}$ \\
As dma8epa but using the EU definition of the daily $8 \mathrm{~h}$ window starting from 17:00 LT of the previous day. \\
(dma8epa: daily maximum $8 \mathrm{~h}$ average statistics according to the US EPA definition. The $8 \mathrm{~h}$ averages are calculated \\
for 24 bins starting at $00: 00 \mathrm{LT}$. The $8 \mathrm{~h}$ running mean for a particular hour is calculated on the concentration for \\
that hour plus the following $7 \mathrm{~h}$. If less than $75 \%$ of data are present (i.e. less than $6 \mathrm{~h}$ ), the average is considered \\
missing. Note that in contrast to the official EPA definition, a daily value is considered valid if at least one $8 \mathrm{~h}$ \\
average is valid.) \\
$\begin{array}{l}\text { Daily maximum value. No data capture criterion is applied; i.e. a daily maximum is valid if at least one hourly value } \\
\text { of the day is present. }\end{array}$ \\
maximum
\end{tabular}

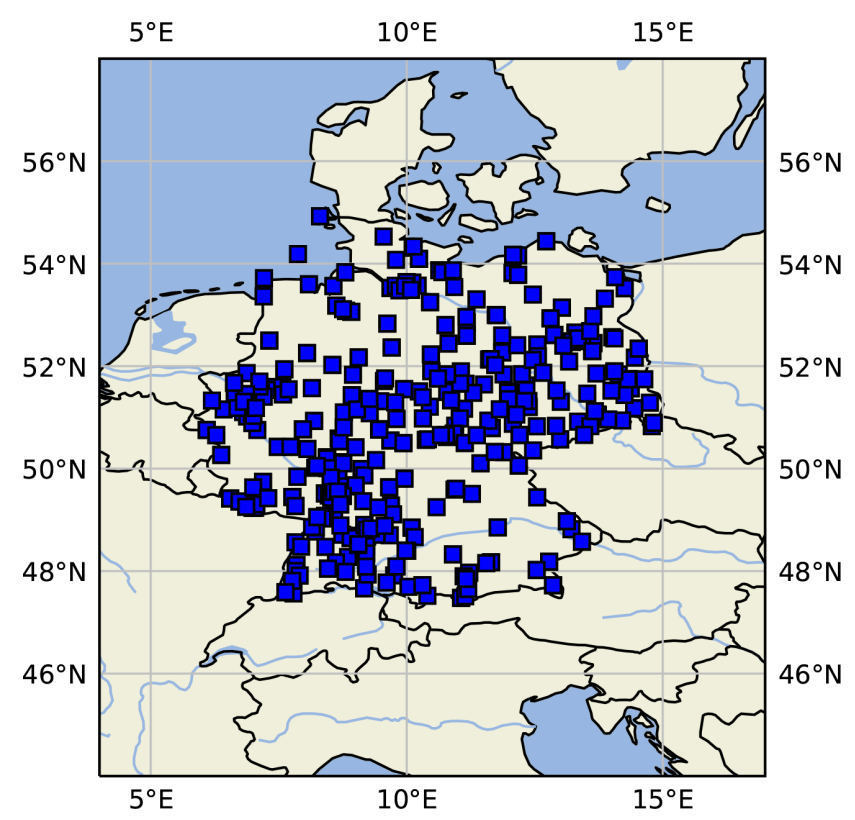

Figure 1. Map of central Europe showing the location of German measurement sites used in this study. This figure was created with Cartopy (Met Office, 2010-2015). Map data @ OpenStreetMap contributors.

\subsection{Data processing}

We split the individual station time series into three nonoverlapping time periods for training, validation, and testing which we will refer to as "set" from now on (see Fig. 2). We only used stations which at least have 1 year of valid data in one set. Firstly, the time span of the training dataset is ranging from 1 January 1997 to 31 December 2007. Secondly, the validation set is ranging from 1 January 2008 to 31 December 2009. Thirdly, the test set ranges from 1 January 2010 to 31 December 2015.

Due to changes in the measurement network over time, the number of stations in the three datasets differ: training data comprise 312 stations, validation data 211 stations, and testing 203 stations. This is by far the largest air quality time series dataset that has been used in a machine learning study so far (see Table 1).

Supervised learning techniques require input data $(\mathbf{X})$ and a corresponding label $(\boldsymbol{y})$ which we create for each station of the three sets as depicted in Algorithm 1.

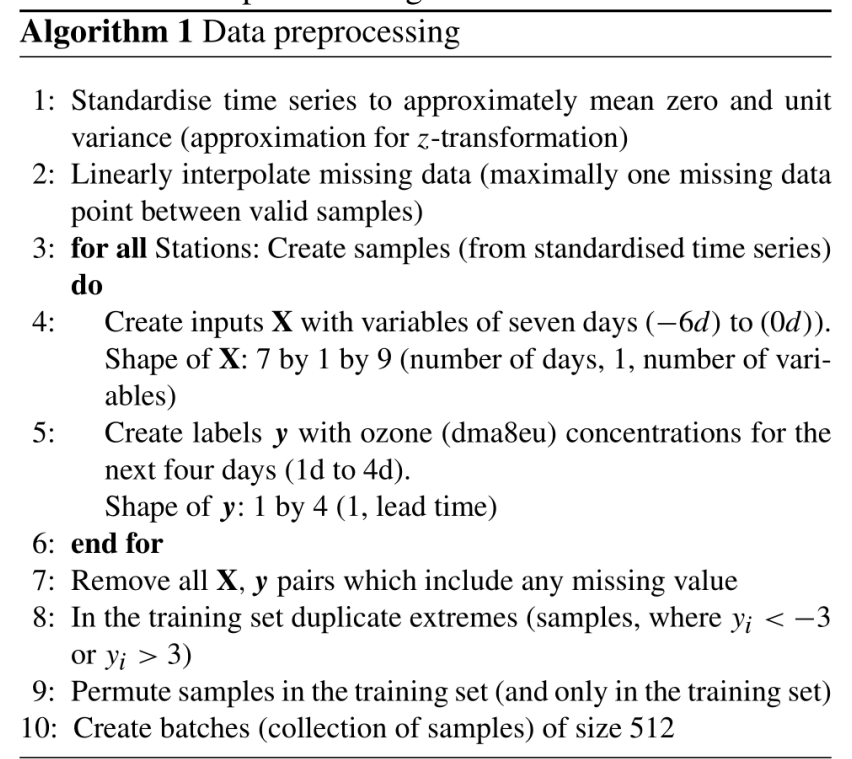

Samples within the same dataset (train, validation, and test) can overlap which means that one missing data point would appear up to seven times in the inputs $\mathbf{X}$ and up to four times in the labels $\boldsymbol{y}$. Consequently, one missing value will cause the removal of 11 samples (algorithm 1, line 7). As we want to keep the number of samples as high as possible, we decided to linearly interpolate (algorithm 1, line 2) the time series if only one consecutive value is missing. Table 4 shows the number of stations per dataset (train, val, test) and the corresponding amount of samples (pairs of inputs $\mathbf{X}$ and labels $\boldsymbol{y}$ ) per dataset. Moreover, Table A1 summarises all 


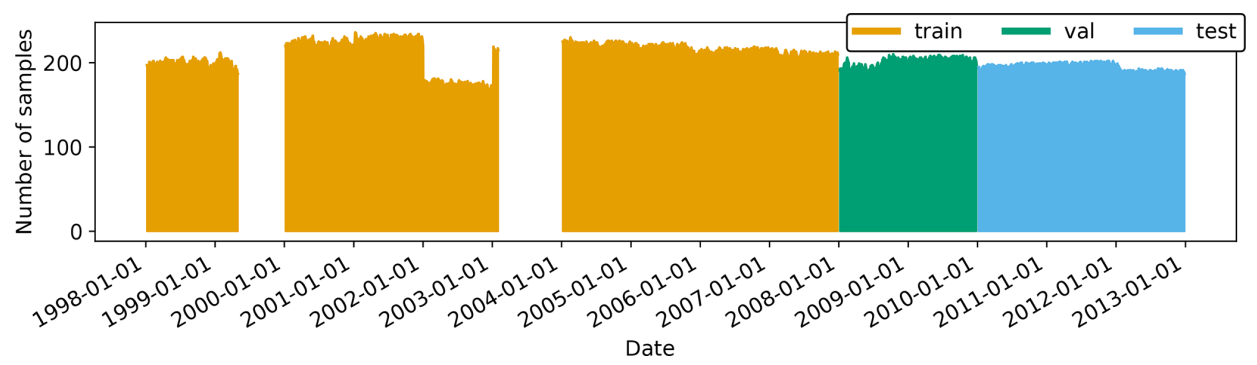

Figure 2. Data availability diagram combined for all variables and all stations. The training set is coloured in orange, the validation set in green, and the test set in blue. Gaps in 1999 and 2003 are caused by missing model data in the TOAR database.

samples per station individually. Figure 1 shows a map of all station locations.

We trained the neural network (details on the network architecture are given in Sect. 3) with data of the training set and tuned hyperparameters exclusively on the validation dataset. For the final analysis and model evaluation, we use the independent test dataset, which was neither used for training the models parameters, nor for tuning the hyperparameters. Random sampling, as is often done in other machine learning applications, and occasionally even in other air quality or weather applications of machine learning, would lead to overly optimistic results due to the multiday auto-correlation of air quality and meteorological time series. Horowitz and Barakat (1979) already pointed to this issue when dealing with statistical tests. Likewise, the alternative split of the dataset into spatially segregated data could lead to the undesired effect that two or more neighbouring stations with high correlation between several paired variables fall into different datasets. Again, this would result in overly optimistic model results.

By applying a temporal split, we ensure that the training data do not directly influence the validation and test datasets. Therefore, the final results reflect the true generalisation capability of our forecasting model.

In accordance with other studies, our initial deep learning experiments with a subset of this data have shown that neural networks, just as other classical regression techniques, have a tendency to focus on the mean of the distribution and perform poorly on the extremes. However, especially the high concentration events are crucial in the air quality context due to their strong impact on human health and the adverse crop effects. Extreme values occur relatively seldomly in the dataset, and it is therefore difficult for the model to learn their associated patterns correctly. To increase the total number of values on the tails of the distribution during training, we append all samples where the standardised label (i.e. the normalised ozone concentration) is $<-3$ or $>3$ for a second time on the training dataset (algorithm 1, line 8).

We selected a batch size of 512 samples (algorithm 1, line 10), because this size is a good compromise between minimising the loss function and optimising computing time per trained epoch. Experiments with larger and smaller batch sizes did not yield significantly different results. Before creating the different training batches, we permute the ordering of samples per station in the training set to ensure that the distribution of each batch is similar to those of the full training dataset (algorithm 1, line 9). Otherwise, each batch would have an under-represented season and consequently would lead to undesired looping during training (e.g. no winter values in the first batch, no autumn values in the second batch).

\section{Model setup}

Our machine learning model is based on a convolutional layer neural network (LeCun et al., 1998), which was initially designed for pattern recognition in computer vision applications. It has been shown that such model architectures work equally well on time series data as other neural network architectures, which have been especially designed for this purpose, such as recurrent neural networks or LSTMs (Dauphin et al., 2017; Bai et al., 2018). Schmidhuber (2015) provides a historical review on different deep learning methods, while Ismail Fawaz et al. (2019) focus especially on deep neural networks for time series.

Our neural network named IntelliO3-ts, version 1.0, primarily consists of two inception blocks (Szegedy et al., 2015), which combine multiple convolutions, execute them in parallel, and concatenate all outputs in the last layer of each block. Figure A2 depicts one inception block including the first input layer, while Figs. A2 and A3 together show the entire model architecture including the final flat and output layers. We treat each input variable (see Table 2) as an individual channel (like R, G, B in images) and use time as the first dimension (this would correspond to the width axis of an image). Inputs (X) consist of the variable values from $7 \mathrm{~d}(-6$ to $0 \mathrm{~d}$ ). Outputs are ozone concentration forecasts (dma8eu) for lead times up to $4 \mathrm{~d}$ ( 1 to $4 \mathrm{~d}$ ). Therefore, we change the kernel sizes in the inception blocks from $1 \times 1,3 \times 3$, and $5 \times 5$, as originally proposed by Szegedy et al. (2015), to $1 \times 1,3 \times 1$, and $5 \times 1$. This allows the network to focus on different temporal relations. The $1 \times 1$ convolutions are also used for information compression (reduction of the number of filters) before larger convolutional kernels are applied (see 
Table 4. Number of stations, total number of samples (pairs of $\mathbf{X}$ and $\boldsymbol{y}$ ), and various statistics of number of samples per station in the training, validation, and test datasets. The number of stations per set varies as not all stations have data through the full period (see Table A1 for details).

\begin{tabular}{lrrrrrrrrrrrrr}
\hline & No. of stations & No. of samples & Mean & SD & Min & $5 \%$ & $10 \%$ & $25 \%$ & $50 \%$ & $75 \%$ & $90 \%$ & $95 \%$ & Max \\
\hline Training & 312 & 643788 & 2063 & 802 & 369 & 668 & 939 & 1426 & 2191 & 2902 & 2989 & 3011 & 3045 \\
Validation & 211 & 145030 & 687 & 61 & 370 & 532 & 625 & 690 & 710 & 721 & 721 & 721 & 721 \\
Test & 203 & 212093 & 1044 & 92 & 466 & 759 & 983 & 1056 & 1075 & 1086 & 1086 & 1086 & 1086 \\
\hline
\end{tabular}

Szegedy et al., 2015). This decreases the computational costs for training and evaluating the network. In order to conserve the initial input shape of the first dimension (time), we apply symmetric padding to minimise the introduction of artefacts related to the borders.

While the original proposed concept of inception blocks has one max-pooling tower alongside the different convolution stacks, we added a second pooling tower, which calculates the average on a kernel size of $3 \times 1$. Thus, one inception block consists of three convolutional towers and two pooling (mean and max) towers. A tower is defined as a collection or stack of successive layers. The outputs of these towers are concatenated in the last layer of an inception block (see Fig. A2). Between individual inception blocks, we added dropout layers (Srivastava et al., 2014) with a dropout rate of 0.35 to improve the network's generalisation capability and prevent overfitting.

Moreover, we use batch normalisation layers (Ioffe and Szegedy, 2015) between each main convolution and activation layer to accelerate the training process (Fig. A2). Those normalisations ensure that for each batch the mean activation is zero with standard deviation of 1 . As proposed in Szegedy et al. (2015), the network has an additional minor tail which helps to eliminate the vanishing gradient problem. Additionally, the minor tail helps to spread the internal representation of data as it strongly penalises large errors.

The loss function for the main tail is the mean squared error:

$L_{\text {main }}=\frac{1}{n} \sum_{i}\left(y_{i, \text { true }}-y_{i, \text { pred }}\right)^{2}$,

while the loss function of the minor tail is

$L_{\text {minor }}=\frac{1}{n} \sum_{i}\left(\left|y_{i, \text { true }}-y_{i, \text { pred }}\right|\right)^{4}$.

All activation functions are exponential linear units (ELUs) (Clevert et al., 2016); only the final output activations are linear (minor and main tail).

The network is built with Keras 2.2.4 (Chollet, 2015) and uses TensorFlow 1.13.1 (Abadi et al., 2015) as the backend. We use Adam (Kingma and Ba, 2014) as optimiser and apply an initial learning rate of $10^{-4}$ (see also Sect. A5).

We train the model for 300 epochs on the Jülich Wizard for European Leadership Science (JUWELS; Jülich Supercomputing Centre, 2019) high-performance computing (HPC) system which is operated by the Jülich Supercomputing Centre (see also Sect. A4 for further details regarding the software and hardware configurations).

\section{Evaluation metrics and reference models}

In general, one can interpret a supervised machine learning approach as an attempt to find an unknown function $\varphi$ which maps an input pattern $(\mathbf{X})$ to the corresponding labels or the ground truth $(\boldsymbol{y})$. The machine learning model is consequently an estimator $(\hat{\varphi})$ which maps $\mathbf{X}$ to an estimate $\hat{\boldsymbol{y}}$ of the ground truth. The goodness of the estimate is quantified by an error function, which the network tries to minimise during training. As the network is only an estimator of the true function, the mapping generally differs:

$\varphi(\mathbf{X})=\boldsymbol{y} \neq \hat{\boldsymbol{y}}=\hat{\varphi}(\mathbf{X})$.

To evaluate the genuine added value of any meteorological or air quality forecasting model, it is essential to apply proper statistical metrics. The following section describes the verification tools, which are used in this study. We provide additional information on joint distributions as introduced by Murphy and Winkler (1987) in Sect. A2.

\subsection{Scores and skill scores}

To quantify a model's informational content, scores like the mean squared error (Eq. 5) are defined to provide an absolute performance measure, while skill scores provide a relative performance measure related to a reference forecast (Eq. 6).

$\operatorname{MSE}(\boldsymbol{m}, \boldsymbol{o})=\frac{1}{N} \sum_{i=1}^{N}\left(m_{i}-o_{i}\right)^{2} \geq 0$

Here, $N$ is the number of forecast-observation pairs, $\boldsymbol{m}$ is a vector containing all model forecasts, and $\boldsymbol{o}$ is a vector containing the observations (Murphy, 1988).

A skill score $S$ may be interpreted as the percentage of improvement of $A$ over the reference $A_{\text {ref. }}$. Its general form is

$S=\frac{A-A_{\text {ref }}}{A_{\text {perf }}-A_{\text {ref }}}$.

Here, $A$ represents a general score, $A_{\text {ref }}$ is the reference score, and $A_{\text {perf }}$ the perfect score. 
For $A=A_{\text {ref }}, S$ becomes zero, indicating that the forecast of interest has no improvements over the reference forecast. A value of $S>0$ indicates an improvement over the reference, while $S<0$ indicates a deterioration. The informative value of a skill score strongly depends on the selected reference forecast. In the case of the mean squared error (Eq. 5), the perfect score is equal to zero and Eq. (6) reduces to

$S(\boldsymbol{m}, \boldsymbol{r}, \boldsymbol{o})=1-\frac{\operatorname{MSE}(\boldsymbol{m}, \boldsymbol{o})}{\operatorname{MSE}(\boldsymbol{r}, \boldsymbol{o})}$,

where $\boldsymbol{r}$ is a vector containing the reference forecast.

\subsection{Reference models}

We used three different reference models: persistence, climatology, and an ordinary least-square model (linear regression). For the climatological reference, we create four subreference models (see Sect. 4.2.2). In the following, we introduce our basic reference models.

\subsubsection{Persistence model}

One of the most straightforward models to build, which in general has good forecast skills on short lead times, is a persistence model. Today's observation of ozone dma8eu concentration is also the prediction for the next $4 \mathrm{~d}$. Obviously, the skill of persistence decreases with increasing lead time. The good performance on short lead times is mainly due to the facts that weather conditions influencing ozone concentrations generally do not change rapidly, and that the chemical lifetime of ozone is long enough.

\subsubsection{Climatological reference models}

We create four different climatological reference models (Case I to Case IV), which are based on the climatology of observations by following Murphy (1988) (also with respect to their terminology, which means that the reference score $A_{\text {ref }}$ is calculated by using the reference forecast $\boldsymbol{r}$ ).

The first reference forecast $\left(A_{\text {ref }}: \boldsymbol{r}=\overline{\boldsymbol{o}}\right.$, Case I) is the internal single value climatology which is the mean of all observations during the test period. This unique value is then applied as reference for all forecasts. As this forecast has only one constant value which is the expectation value, this reference model is well calibrated but not refined at all.

The second reference $\left(A_{\text {ref }}: \boldsymbol{r}=\boldsymbol{o}^{*}\right.$, Case II) is an internal multi-value climatology. Here, we calculate 12 arithmetic means, where each of the means is the monthly mean over all years in the test set (one mean for all Januaries from 2012 to 2015, one for all Februaries, etc.). The corresponding monthly mean is applied as reference. Therefore, Case II allows testing if the model has skill in reproducing the seasonal cycle of the observations.

The third reference $\left(A_{\text {ref }}: \boldsymbol{r}=\overline{\boldsymbol{\mu}}\right.$, Case III) is an external single value climatology which is the mean of all available observations during the training and validation periods. This reference does not include any direct information on the test set. Therefore, one can access the information if the forecast of interest captures features which are not directly present in the training and validation set.

Finally, the fourth reference $\left(A_{\text {ref }}: \boldsymbol{r}=\boldsymbol{\mu}^{*}\right.$, Case IV) is an external multi-value climatology. A tabular summary explaining the individual formulae and terms following Murphy (1988) is given in Sect. A3. The last two references are calculated on a much longer time series than the first ones.

\subsubsection{OLS reference model}

The third reference model is an ordinary least-square (OLS) model. We train the OLS model by using the statsmodels package v0.10 (Seabold and Perktold, 2010). The OLS model is trained on the same data as the neural network (training set) and serves as a linear competitor.

\section{Results}

As described in Sect. 3, we split our data into three subsets (training, validation, and test set). We only used the independent test dataset to evaluate the forecasting capabilities of the IntelliO3-ts network. During training and hyperparameter optimisation, only the training and validation sets were used, respectively. Therefore, the following results reflect the true generalisation capability of IntelliO3-ts. Before discussing the results in detail below, we would like to point out again that this is the first time that one neural network has been trained to forecast data from an entire national air quality monitoring station network. Also, the network has been trained exclusively with time series data from air pollutant measurements and a meteorological reanalysis. No additional information, such as geographic coordinates, or other hints that could be used by the network to perform a station classification task, has been used. The impact of such extra information will be the subject of another study.

\subsection{Forecasting results}

Figure 3 shows the observed monthly $\mathrm{O}_{3}$-dma8eu distribution (green) and the corresponding network predictions for a lead time of up to $4 \mathrm{~d}$ (dark to light blue) summarised for all 203 stations in the test set. The network clearly captures the seasonal cycle. Nonetheless, the arithmetic mean (black triangles) and the median tend to shift towards their respective annual mean with increasing lead time (see also Fig. 6). In spring and autumn, the observed and forecasted distributions match well, while in summer and wintertime the network underestimates the interquartile range (IQR) and does not reproduce the extremes values (for example, the maxima in July/August or the minima in December/January/February). 


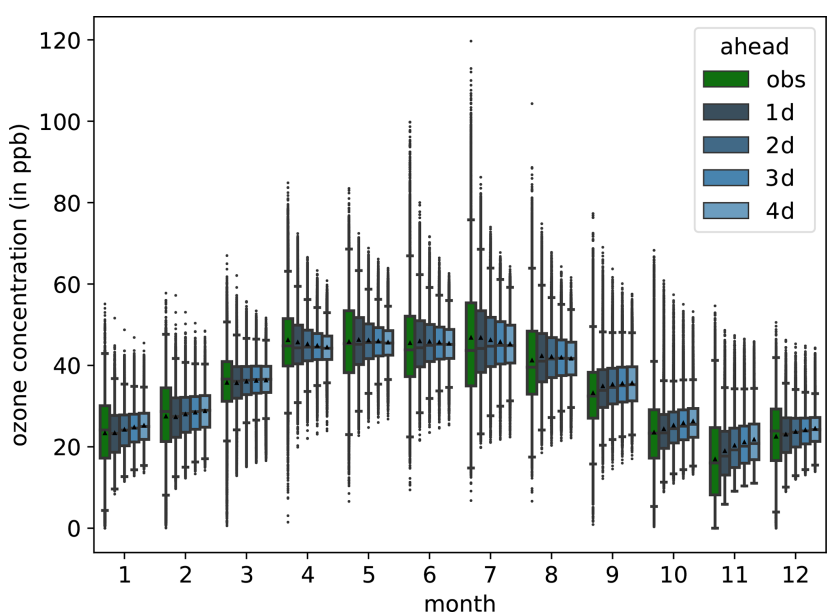

Figure 3. Monthly dma8eu ozone concentrations for all test stations as boxplots. Measurements are denoted by "obs" (green), while the forecasts are denoted by " $1 \mathrm{~d}$ " (dark blue) to " $4 \mathrm{~d}$ " (light blue). Whiskers have a maximal length of one interquartile range. The black triangles denote the arithmetic means.

\subsection{Comparison with competitive models}

The skill scores based on the mean squared error (MSE) evaluated over all stations in the test set are summarised in Fig. 4. In the left and centre groups of boxes and whiskers, the IntelliO3-ts model (labelled "CNN") and the OLS model are compared against persistence as reference. The right group of boxes and whiskers shows the comparison between IntelliO3-ts and OLS. The mean skill score for IntelliO3-ts against persistence is positive and increases with time. The OLS forecast shows similar behaviour in terms of its temporal evolution but exhibits a slightly lower skill score throughout the $4 \mathrm{~d}$ forecasting period. The increases in skill score in both cases is mainly due to the decreasing performance of the persistence model (see also Sect. 4.2.1). Consequently, IntelliO3-ts shows a positive skill score when the OLS model is used as a reference, indicating a small genuine added value over the OLS model.

In comparison with climatological reference forecasts as introduced in Sect. 4.1 and summarised in Table A2, the skill scores are high for the first lead time (1d) and decrease with increasing lead time (Fig. 5). Both cases with a single value as reference (internal Case I, external Case III) maintain a skill score above 0.4 over the $4 \mathrm{~d}$. These high skill scores are a direct result of the fact that IntelliO3-ts captures the seasonal cycle as shown in Fig. 3, while the reference forecasts only report the overall mean as a single value prediction.

If the reference includes the seasonal variation (Case II and Case IV), the IntelliO3-ts skill score is still better than 0.4 for the first day ( $1 \mathrm{~d})$, but then it decreases rapidly and even becomes negative on day 4 for Case II. The skill scores for Case II are lower than for Case IV as the reference climatology (i.e. the monthly mean values) is calculated on the

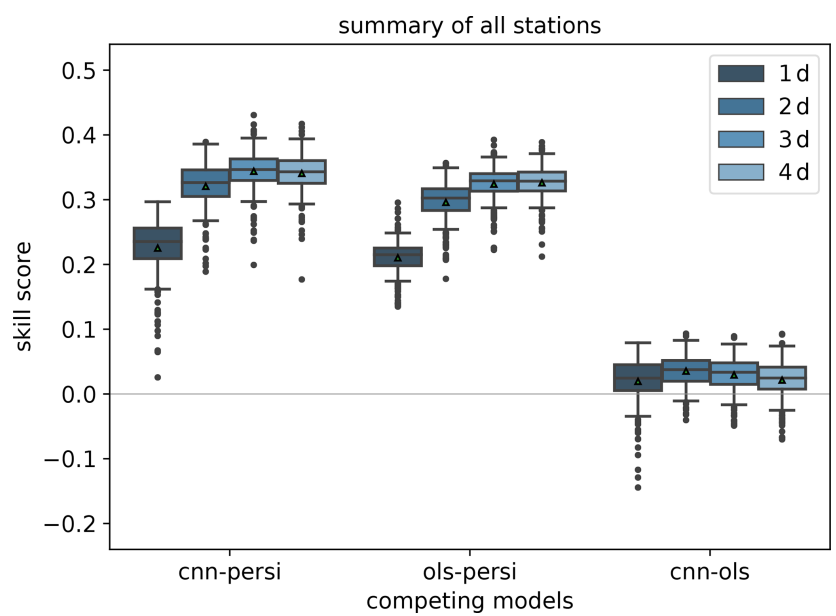

Figure 4. Skill scores of the IntelliO3-ts (cnn) versus the two reference models' persistence (persi) and ordinary least square (ols) based on the mean squared error; separated for all lead times $(1 \mathrm{~d}$ (dark blue) to $4 \mathrm{~d}$ (light blue)). Positive values denote that the first mentioned prediction model performs better than the reference model (mentioned as second). The triangles denote the arithmetic means.

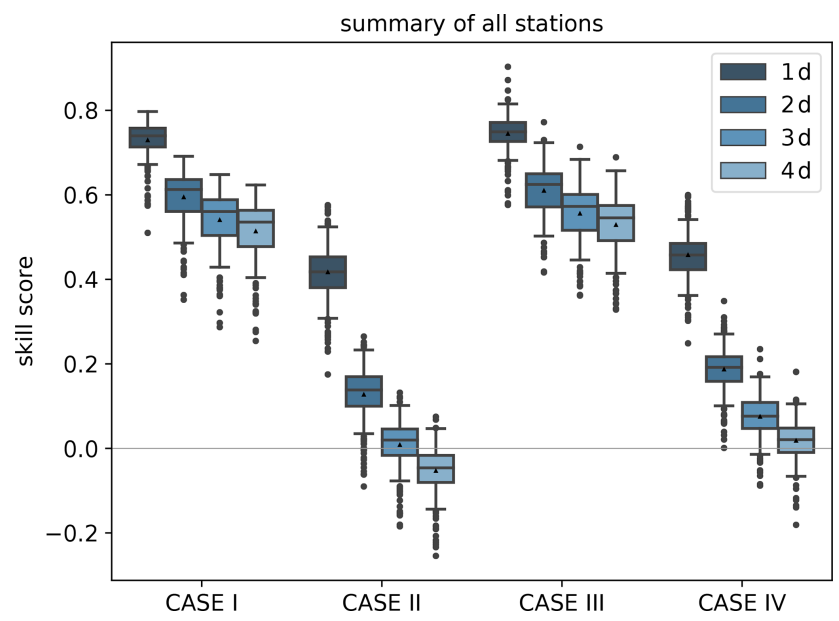

Figure 5. Skill scores of IntelliO3-ts with respect to climatological reference forecasts: with internal single value reference (Case I), internal multi-value (monthly) reference (Case II), external single (Case III), and external multi-value (monthly) reference (Case IV) for all lead times from $1 \mathrm{~d}$ (dark blue) to $4 \mathrm{~d}$ (light blue). Triangles denote the arithmetic means.

test set itself. These results show that, for the vast majority of stations, our model performs much better than a seasonal climatology for a $1 \mathrm{~d}$ forecast, and it is still substantially better than the climatology after $2 \mathrm{~d}$. However, there are some stations which yield a negative skill score even on day 2 in the Case II comparison. Longer-term forecasts with this model setup do not add value compared to the computationally much cheaper monthly mean climatological forecast. 


\subsection{Analysis of joint distributions}

The full joint distribution in terms of calibration refinement factorisation (Sect. A2) is shown in Fig. 6a (first lead time; $1 \mathrm{~d}$ ) to $\mathrm{d}$ (last lead time; $4 \mathrm{~d}$ ). The marginal distribution (refinement) is shown as histogram (light grey; sample size), while the conditional distribution (calibration) is presented by specific percentiles in different line styles. If the median (0.5th quantile, solid line) is below the reference, the network exhibits a high bias with respect to the observations, and vice versa. Obviously, quantiles in value regions with many data samples are more robust and therefore more credible than quantiles in data-sparse concentration regimes (Murphy et al., 1989). On the first lead time (d1; Fig. 6), the IntelliO3-ts network has a tendency to slightly overpredict concentrations $\lesssim 30 \mathrm{ppb}$. On the other hand, the forecast underestimates concentrations above $\gtrsim 70 \mathrm{ppb}$.

Both very high and very low forecasts are rare (note the logarithmic axis for the sample size). Therefore, the results in these regimes have to be treated with caution. Further detail is provided in Fig. A1, where the conditional biases are shown (terms BI, BII, and BIV in Sect. A3) which decrease the maximal climatological potential skill score (term AI; see also Table A2).

With increasing lead time, the model looses its capability to predict concentrations close to zero and high concentrations above $80 \mathrm{ppb}$. The marginal distribution develops a pronounced bimodal shape which is directly linked to the conditional biases. The number of high (extreme) ozone concentrations is relatively low, resulting in few training examples. The network tries to optimise the loss function with respect to the most common values. As a result, predictions of concentrations near the mean value of the distribution are generally more correct than predictions of values from the fringes of the distribution. Moreover, this also explains why the model does not perform substantially better than the monthly mean climatology forecasts (Case II, Case IV). This problem also becomes apparent in other studies. For example, Sayeed et al. (2020) focus their categorical analysis on a threshold value of $55 \mathrm{ppbv}$ (maximum $8 \mathrm{~h}$ average) which corresponds to the air quality index value "moderate" (AQI 51 to 100), instead of the legal threshold value of $70 \mathrm{ppbv}$ (U.S. Environmental Protection Agency, 2016, Table 5, therein), as the model shows better skills in this regime.

To shed more light on the factors influencing the forecast quality, we analyse the network performance individually for each season (DJF, MAM, JJA, and SON). Conditional quantile plots for individual seasons can be found in the Appendix (Sect. A6). As mentioned above, the bimodal shape of the marginal distribution is mainly caused by the network's weakness to predict very high and low ozone concentrations. Moreover, the seasonal decomposition shows that the left mode is caused by the fall (SON) and winter (DJF) seasons (Figs. A7a-d and A4a-d). In both seasons, the most common values fall into the same concentration range, while the right tail of SON is much more pronounced than for DJF, with higher values occurring primarily in September. In the summer season (JJA, Fig. A6a-d), the most frequently predicted values correspond to the location of the right mode of Fig. 6a-d. During DJF, MAM, and JJA, the model has a stronger tendency of under-forecasting with increasing lead time (median line moves above the reference line).

\subsection{Relevance of input variables}

To analyse the impact of individual input variables on the forecast results, we apply a bootstrapping technique as follows: we take the original input of one station, keep eight of the nine variables unaltered, and randomly draw (with replacement) the missing variable (20 times per variable per station). This destroys the temporal structure of this specific variable so that the network will no longer be able to use this information for forecasting. Compared to alternative approaches, such as re-training the model with fewer input variables, setting all variable values to zero, etc., this method has two main advantages: (i) the model does not need to be retrained, and thus the evaluation occurs with the exact same weights that were learned from the full dataset, and (ii) the distribution of the input variable remains unchanged so that adverse effects, for example, due to correlated input variables, are excluded. However, we note that this method may underestimate the impact of a specific variable in the case of correlated input data, because in such cases the network will focus on the dominant feature (here ozone). Also, this analyses only evaluates the behaviour of the deep learning model and does not evaluate the impact of these variables on actual ozone formation in the atmosphere.

After the randomisation of one variable, we apply the trained model on this modified input data and compare the new prediction with the original one. For comparison, we apply the skill score (Eq. 6) based on the MSE where we use the original forecast as a reference. Consequently, the skill score will be negative if the bootstrapped variable has a significant impact on model performance. Figure 7 shows the skill scores for all variables ( $x$ axis) and lead times (dark ( $1 \mathrm{~d})$ to light blue ( $4 \mathrm{~d}$ ) boxplots). Ozone is the most crucial input variable, as it has by far the lowest skill score for all lead times. With increasing lead time, the skill score increases but stays lower than for any other variable. In contrast, the model does not derive much skill from the variables nitrogen oxide, nitrogen dioxide, and the planetary boundary layer height. In other words, the network does not perform worse, when randomly drawn values replace one of those original time series. Relative humidity, temperature, and the wind's $u$ component have an impact on the model performance. With increasing lead time, these influences decrease. 


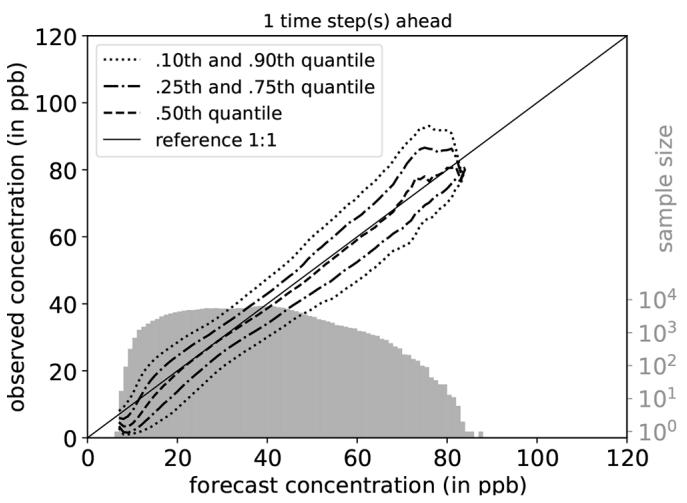

(a)

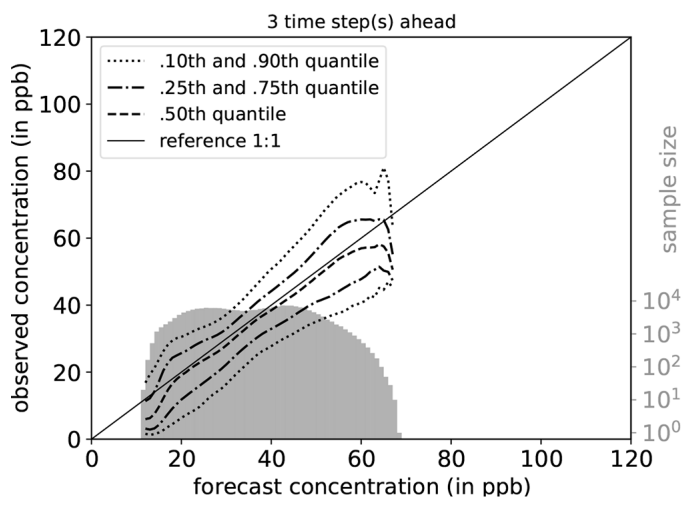

(c)

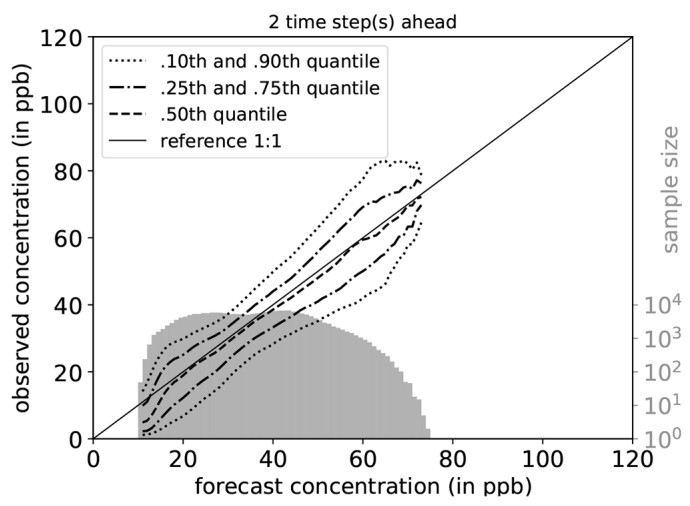

(b)

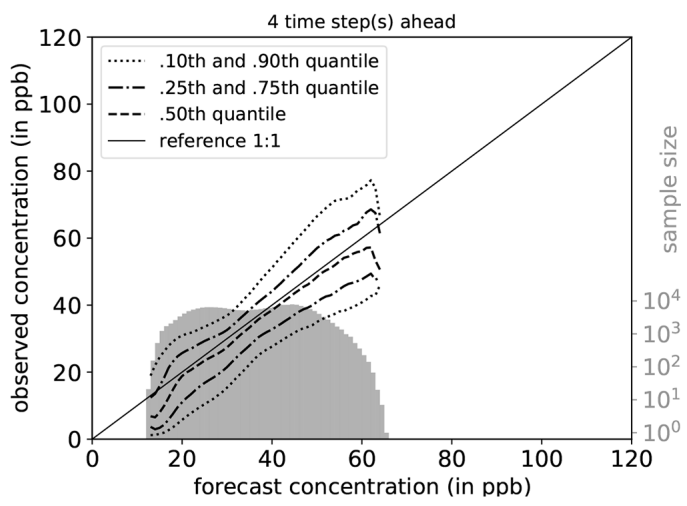

(d)

Figure 6. Conditional quantile plot for all IntelliO3-ts predictions for a lead time of $1 \mathrm{~d}(\mathbf{a}), 2 \mathrm{~d}$ (b), $3 \mathrm{~d}$ (c), and $4 \mathrm{~d}$ (d). Conditional percentiles (0.10th and 0.90th, 0.25th and 0.75th and 0.50th) from the conditional distribution $f\left(o_{j} \mid m_{i}\right)$ are shown as lines in different styles. The reference line indicates a hypothetical perfect forecast. The marginal distribution of the forecast $f\left(m_{i}\right)$ is shown as log histogram (right axis, light grey). All calculations are done by using a bin size of $1 \mathrm{ppb}$. Quantiles are smoothed by using a rolling mean of a window size of 3. (After Murphy et al., 1989.)

\section{Limitations and additional remarks}

Even though IntelliO3-ts v1.0 generalises well on an unseen testing set (see Sect. 5), it still has some limitations related to the applied data split.

By splitting the data into three consecutive, nonoverlapping sets, we ensure that the datasets are as independent as possible. On the other hand, this independence comes at the cost that changes of trends in the input variables may not be captured, especially as our input data are not detrended. Indeed, at European non-urban measurement sites, several ozone metrics related to high concentrations (e.g. fourth highest daily maximum $8 \mathrm{~h}$ (4MDA8) or the 95th percentile of hourly concentrations) show a significant decrease during our study period (1997 to 2015) (Fleming et al., 2018; Yan et al., 2018). Our data-splitting method for evaluating the generalisation capability is conservative in the sense that we evaluate the model on the test set, which has the largest possible distance to the training set. If our research model shall be transformed into an operational system, we suggest applying online learning and use the latest available data for subsequent training cycles (see, for example, Sayeed et al., 2020).

\section{Conclusions}

In this study, we developed and evaluated IntelliO3-ts, a deep learning forecasting model for daily near-surface ozone concentrations (dma8eu) at arbitrary air quality monitoring stations in Germany. The model uses chemical $\left(\mathrm{O}_{3}, \mathrm{NO}, \mathrm{NO}_{2}\right)$ and meteorological time series of the previous $6 \mathrm{~d}$ to create forecasts for up to $4 \mathrm{~d}$ into the future. IntelliO3-ts is based on convolutional inception blocks, which allow us to calculate concurrent convolutions with different kernel sizes. The model has been trained on 10 years of data from 312 background stations in Germany. Hyperparameter tuning and 


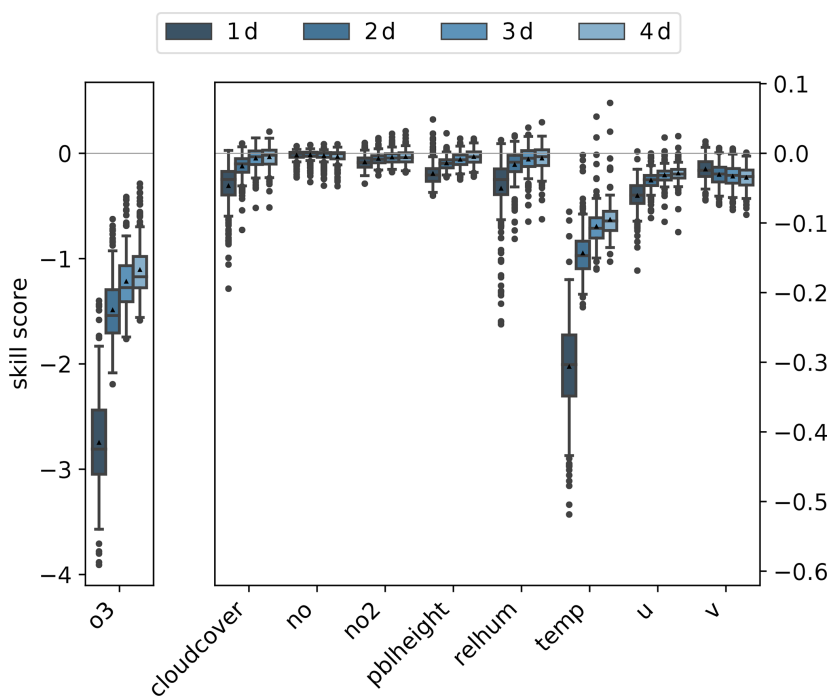

Figure 7. Skill scores of bootstrapped model predictions having the original forecast as the reference model are shown as boxplots for all lead times from $1 \mathrm{~d}$ (dark blue) to $4 \mathrm{~d}$ (light blue). The skill score for ozone is shown on the left $y$ axis, while the skill score of the other variables is shown on the right $y$ axis.

model evaluation were done with independent datasets of 2 and 6 years length, respectively.

The model generalises well and generates good quality forecasts for lead times up to $2 \mathrm{~d}$. These forecasts are superior compared to the reference persistence, ordinary least squares, annual, and seasonal climatology models. After $2 \mathrm{~d}$, the forecast quality degrades, and the forecast adds no value compared to a monthly mean climatology of dma8eu ozone levels. We could primarily attribute this to the network's tendency to converge to the mean monthly value. The model does not have any spatial context information which could counteract this tendency. Near-surface ozone concentrations at background stations are highly influenced by air mass advection, but the IntelliO3-ts network has no way of taking upwind information into account yet. We will investigate spatial context approaches in a forthcoming study.

We observed that the model loses refinement with increasing lead time which results in unsatisfactory predictions on the tails of the observed ozone concentration. We were able to attribute this weakness to the under-representation of extreme (either very small or high) levels in the training dataset. This is a general problem for machine learning applications and regression methods. The machine learning community is investigating possible solutions to lessen the impact of such data imbalances, but their adaptation is beyond the scope of this paper as proposed techniques are not directly applicable to those time series (auto-correlation time).

Bootstrapping individual time series of the input data to analyse the importance of those variables on the predictive skill showed that the model mainly focused on the previous ozone concentrations. Temperature and relative humidity only have a small effect on the model performance, while the time series of $\mathrm{NO}, \mathrm{NO}_{2}$, and planetary boundary layer (PBL) have no impact.

The IntelliO3-ts network extends previous work by using a new network architecture, and training one model on a much larger set of measurement station data and longer time periods. In light of Rasp and Lerch (2018), who used several neural networks to postprocess ensemble weather forecasts, we applied meteorological evaluation metrics to perform a pointby-point comparison, which is not common in the field of deep learning. We hope that the forecast quality of IntelliO3ts can be further improved if we take spatial context information into account so that the advection of background ozone and ozone precursors can be learned by the model. 


\section{Appendix A}

\section{A1 Information on used stations}

Table A1 lists all measurement stations which we used in this study. The table also shows the number of samples $(\mathbf{X}, \boldsymbol{y})$ for each of the three datasets (training, validation, and test).

Table A1. Number of samples (input and output pairs) per station separated by training, validation (val), and test dataset. "-" denotes no samples in a set.

\begin{tabular}{|c|c|c|c|}
\hline & Training & Val & Test \\
\hline \multicolumn{4}{|l|}{ Stat. ID } \\
\hline DEBB001 & 1104 & - & - \\
\hline DEBB006 & 1455 & - & - \\
\hline DEBB007 & - & 721 & 1086 \\
\hline DEBB009 & 1438 & - & - \\
\hline DEBB021 & 2512 & 705 & 1052 \\
\hline DEBB024 & 2592 & - & - \\
\hline DEBB028 & 1353 & - & - \\
\hline DEBB031 & 2577 & - & - \\
\hline DEBB036 & 1008 & - & - \\
\hline DEBB038 & 1245 & - & - \\
\hline DEBB040 & 760 & - & - \\
\hline DEBB042 & 2902 & 721 & 1086 \\
\hline DEBB043 & 2194 & - & - \\
\hline DEBB048 & 2473 & 721 & 1075 \\
\hline DEBB050 & 2510 & 721 & - \\
\hline DEBB051 & 1006 & - & - \\
\hline DEBB053 & 2115 & 706 & 1086 \\
\hline DEBB055 & 1887 & 721 & 1053 \\
\hline DEBB063 & 1392 & 721 & 1086 \\
\hline DEBB064 & 1480 & 721 & 1086 \\
\hline DEBB065 & 1411 & 699 & 1086 \\
\hline DEBB066 & 1451 & 721 & 1086 \\
\hline DEBB067 & 1073 & 721 & 1086 \\
\hline DEBB075 & - & 721 & 1079 \\
\hline DEBB0 & - & 622 & 1086 \\
\hline DEBB083 & - & - & 1086 \\
\hline DEBE010 & 1372 & 707 & 1035 \\
\hline DEBE032 & 2441 & 690 & 1060 \\
\hline DEBE034 & 2506 & 671 & 1031 \\
\hline DEBE051 & 2433 & 694 & 1053 \\
\hline DEBE056 & 2481 & 678 & 1055 \\
\hline DEBE062 & 1941 & 677 & 1069 \\
\hline DEBW004 & 1440 & 721 & 1086 \\
\hline DEBW006 & 1451 & 721 & 1086 \\
\hline DEBW007 & 1440 & 699 & - \\
\hline DEBW008 & 656 & - & - \\
\hline DEBW010 & 3041 & 708 & 1086 \\
\hline DEBW013 & 1432 & 710 & 1079 \\
\hline DEBW019 & 2962 & 710 & 1078 \\
\hline DEBW020 & 1520 & - & \\
\hline
\end{tabular}

Table A1. Continued.

\begin{tabular}{lrrr}
\hline & Training & Val & Test \\
\hline Stat. ID & & & \\
\hline DEBW021 & 1579 & - & - \\
DEBW023 & 1430 & 721 & 1078 \\
DEBW024 & 3011 & 713 & 1086 \\
DEBW025 & 1530 & - & - \\
DEBW026 & 3005 & 721 & - \\
DEBW027 & 3005 & 699 & 1075 \\
DEBW028 & 1510 & - & - \\
DEBW029 & 3012 & 721 & 1086 \\
DEBW030 & 2966 & - & - \\
DEBW031 & 2970 & 711 & 1069 \\
DEBW032 & 2648 & - & - \\
DEBW034 & 3045 & 707 & - \\
DEBW035 & 2281 & - & - \\
DEBW036 & 1183 & - & - \\
DEBW037 & 3023 & 721 & - \\
DEBW039 & 2999 & 710 & 1086 \\
DEBW041 & 1581 & - & - \\
DEBW042 & 2617 & 708 & 1067 \\
DEBW044 & 1571 & - & - \\
DEBW045 & 656 & - & - \\
DEBW046 & 2990 & 699 & 1086 \\
DEBW047 & 1563 & - & - \\
DEBW049 & 646 & - & - \\
DEBW050 & 1556 & - & - \\
DEBW052 & 2652 & 721 & 1078 \\
DEBW053 & 1574 & - & - \\
DEBW054 & 1566 & - & - \\
DEBW056 & 2938 & 721 & 1086 \\
DEBW057 & 644 & - & - \\
DEBW059 & 2974 & 721 & 1086 \\
DEBW060 & 1571 & - & - \\
DEBW065 & 1540 & - & - \\
DEBW072 & 480 & - & - \\
DEBW076 & 3035 & 721 & 708 \\
DEBW081 & 2654 & 721 & 1079 \\
DEBW084 & 1444 & 721 & 1042 \\
DEBW087 & 3043 & 713 & 1086 \\
DEBW094 & 2188 & - & - \\
DEBW102 & 1122 & - & - \\
DEBW103 & 2525 & 721 & - \\
DEBW107 & 1801 & 714 & 1086 \\
DEBW110 & 1107 & 721 & - \\
DEBW111 & 1083 & 703 & - \\
DEBW112 & 651 & 721 & 1079 \\
DEBW113 & 678 & - & - \\
DEBY002 & 2924 & 721 & 747 \\
DEBY004 & 2917 & 707 & 1028 \\
DEBY005 & 2959 & 714 & 1086 \\
DEBY013 & 1412 & 652 & 980 \\
DEBY017 & 1250 & - & - \\
\hline & & &
\end{tabular}


Table A1. Continued.

\begin{tabular}{lrrr}
\hline & Training & Val & Test \\
\hline Stat. ID & & & \\
\hline DEBY020 & 2976 & 721 & 1013 \\
DEBY031 & 2927 & 678 & 1072 \\
DEBY032 & 2975 & 721 & 711 \\
DEBY034 & 1555 & - & - \\
DEBY039 & 2554 & 721 & 1067 \\
DEBY047 & 1895 & 721 & 754 \\
DEBY049 & 2918 & 693 & 1066 \\
DEBY052 & 2929 & 708 & 1035 \\
DEBY062 & 1411 & 704 & 748 \\
DEBY072 & 2907 & 690 & 1055 \\
DEBY077 & 1409 & 721 & 724 \\
DEBY079 & 2878 & 721 & 671 \\
DEBY081 & 2932 & 523 & 713 \\
DEBY082 & 1592 & - & - \\
DEBY088 & 2986 & 713 & 1062 \\
DEBY089 & 2644 & 721 & 1086 \\
DEBY092 & 616 & - & - \\
DEBY099 & 1828 & 703 & 725 \\
DEBY109 & 1310 & 713 & 1071 \\
DEBY113 & 1347 & 706 & 1086 \\
DEBY118 & 937 & 705 & 727 \\
DEBY122 & - & - & 877 \\
DEHB001 & 2567 & 710 & 953 \\
DEHB002 & 2287 & 702 & 1053 \\
DEHB003 & 2546 & 695 & - \\
DEHB004 & 1428 & 708 & 1037 \\
DEHB005 & 2518 & 683 & 1066 \\
DEHE001 & 1451 & 721 & 1086 \\
DEHE008 & 2447 & 707 & 1075 \\
DEHE010 & 1600 & - & - \\
DEHE013 & - & 721 & 1086 \\
DEHE017 & 1562 & - & - \\
DEHE018 & 3016 & 721 & 1086 \\
DEHE019 & 1958 & - & - \\
DEHE022 & 2643 & 721 & 1086 \\
DEHE023 & 2966 & 708 & 466 \\
DEHE024 & 2935 & 710 & 1086 \\
DEHE025 & 1554 & - & - \\
DEHE026 & 2877 & 697 & 1086 \\
DEHE027 & 1536 & - & - \\
DEHE028 & 2946 & 710 & 1068 \\
DEHE030 & 3028 & 721 & 1086 \\
DEHE032 & 2926 & 714 & 1075 \\
DEHE033 & 1835 & - & - \\
DEHE034 & 1880 & - & - \\
DEHE039 & 2966 & 721 & 1079 \\
DEHE043 & 3004 & 721 & 1074 \\
DEHE044 & 2543 & 721 & 1086 \\
DEHE045 & 2535 & 699 & 1086 \\
DEHE046 & 2513 & 714 & 1086 \\
\hline & & &
\end{tabular}

Table A1. Continued.

\begin{tabular}{|c|c|c|c|}
\hline & Training & Val & Test \\
\hline \multicolumn{4}{|l|}{ Stat. ID } \\
\hline DEHE048 & 1043 & - & - \\
\hline DEHE050 & 1014 & - & - \\
\hline DEHE051 & 2331 & 721 & 1086 \\
\hline DEHE052 & 2078 & 713 & 1086 \\
\hline DEHE058 & 789 & 721 & 1086 \\
\hline DEHE060 & 704 & 672 & 1086 \\
\hline DEHH008 & 1439 & 721 & 1086 \\
\hline DEHH021 & 2624 & 710 & 1086 \\
\hline DEHH033 & 2148 & 682 & 1067 \\
\hline DEHH047 & 2175 & 696 & 1086 \\
\hline DEHH049 & 2150 & 721 & 1075 \\
\hline DEHH050 & 2131 & 721 & 1069 \\
\hline DEMV001 & 794 & - & - \\
\hline DEMV004 & 2908 & 721 & 1058 \\
\hline DEMV007 & 2986 & 721 & 1053 \\
\hline DEMV012 & 2885 & 710 & 1086 \\
\hline DEMV017 & 2507 & 708 & 1086 \\
\hline DEMV018 & 2113 & 710 & - \\
\hline DEMV019 & 1429 & 706 & 1086 \\
\hline DEMV021 & 600 & 688 & 1072 \\
\hline DEMV024 & - & - & 908 \\
\hline DENI011 & 2611 & 452 & 1086 \\
\hline DENI016 & 3034 & 627 & 1051 \\
\hline DENI019 & 2919 & - & - \\
\hline DENI020 & 2984 & 667 & 1086 \\
\hline DENI028 & 2927 & 516 & 1086 \\
\hline DENI029 & 2903 & 692 & 1086 \\
\hline DENI031 & 1410 & 451 & 1079 \\
\hline DENI038 & 2599 & 573 & 1083 \\
\hline DENI041 & 2935 & 525 & 1086 \\
\hline DENI042 & 2939 & 553 & 1072 \\
\hline DENI043 & 2941 & 606 & 1086 \\
\hline DENI051 & 2976 & - & 1072 \\
\hline DENI052 & 2910 & 529 & 1086 \\
\hline DENI054 & 2997 & 596 & 1086 \\
\hline DENI058 & 2398 & - & 1086 \\
\hline DENI059 & 2408 & 451 & 1079 \\
\hline DENI060 & 2386 & 677 & 1086 \\
\hline DENI062 & 2482 & 625 & 1080 \\
\hline DENI063 & 2385 & 460 & 1073 \\
\hline DENI077 & - & - & 1079 \\
\hline DENW004 & 1148 & - & - \\
\hline DENW006 & 1367 & 694 & 1026 \\
\hline DENW008 & 2511 & 701 & 1064 \\
\hline DENW010 & 1397 & - & - \\
\hline DENW013 & 1830 & - & - \\
\hline DENW015 & 1451 & - & - \\
\hline DENW018 & 1196 & - & - \\
\hline DENW028 & 1655 & - & - \\
\hline DENW029 & 2530 & - & - \\
\hline DENW030 & 2785 & 630 & 998 \\
\hline
\end{tabular}


Table A1. Continued.

\begin{tabular}{|c|c|c|c|}
\hline & Training & Val & Test \\
\hline \multicolumn{4}{|l|}{ Stat. ID } \\
\hline DENW036 & 1314 & - & - \\
\hline DENW038 & 2598 & 652 & 1079 \\
\hline DENW042 & 1185 & - & - \\
\hline DENW047 & 1350 & - & - \\
\hline DENW050 & 2488 & - & - \\
\hline DENW051 & 1206 & - & - \\
\hline DENW053 & 1795 & 678 & 1051 \\
\hline DENW059 & 1777 & 648 & 980 \\
\hline DENW062 & 1078 & - & - \\
\hline DENW063 & 2816 & - & - \\
\hline DENW064 & 2887 & 589 & 1052 \\
\hline DENW065 & 2877 & 550 & 1045 \\
\hline DENW066 & 2865 & - & - \\
\hline DENW067 & 2473 & 686 & 1079 \\
\hline DENW068 & 2892 & 447 & 1009 \\
\hline DENW071 & 1827 & 713 & 1071 \\
\hline DENW078 & 1382 & 681 & 1078 \\
\hline DENW079 & 2040 & 706 & 1086 \\
\hline DENW080 & 2147 & 689 & 1043 \\
\hline DENW081 & 2422 & 646 & 1058 \\
\hline DENW094 & 1980 & 627 & 1058 \\
\hline DENW095 & 1981 & 681 & 1071 \\
\hline DENW096 & 700 & - & - \\
\hline DENW179 & 766 & 699 & 1079 \\
\hline DENW247 & - & 572 & 1066 \\
\hline DERP001 & 1421 & 721 & 1068 \\
\hline DERP007 & 2652 & 721 & 1077 \\
\hline DERP013 & 2883 & 708 & 1067 \\
\hline DERP014 & 2967 & 703 & 1061 \\
\hline DERP015 & 2810 & 710 & 1047 \\
\hline DERP016 & 2962 & 721 & 1086 \\
\hline DERP017 & 2955 & 721 & 1055 \\
\hline DERP019 & 1413 & 708 & 1041 \\
\hline DERP021 & 2996 & 713 & 1025 \\
\hline DERP022 & 2989 & 701 & 1059 \\
\hline DERP025 & 2918 & 691 & 1086 \\
\hline DERP028 & 2802 & 678 & 1026 \\
\hline DESH005 & 962 & - & - \\
\hline DESH006 & 614 & - & - \\
\hline DESH008 & 3031 & 721 & 1053 \\
\hline DESH016 & 2635 & 698 & - \\
\hline DESH021 & 1107 & - & - \\
\hline DESH023 & 1700 & 721 & 1066 \\
\hline DESH033 & - & 721 & 1072 \\
\hline DESL003 & 1393 & 713 & 1086 \\
\hline DESL008 & 1371 & - & - \\
\hline DESL011 & 2776 & 710 & 1086 \\
\hline DESL012 & - & - & 1086 \\
\hline
\end{tabular}

Table A1. Continued.

\begin{tabular}{lrrr}
\hline & Training & Val & Test \\
\hline Stat. ID & & & \\
\hline DESL017 & 2785 & 714 & 1086 \\
DESL018 & 1656 & 710 & 1086 \\
DESL019 & 1371 & 472 & 1057 \\
DESN001 & 2925 & 689 & 1072 \\
DESN004 & 3011 & 705 & 1086 \\
DESN005 & 1166 & - & - \\
DESN011 & 2613 & 694 & 1073 \\
DESN012 & 2995 & 721 & - \\
DESN014 & 2256 & - & - \\
DESN017 & 3028 & 704 & - \\
DESN019 & 2934 & 721 & - \\
DESN024 & 3017 & 721 & - \\
DESN028 & 401 & - & - \\
DESN036 & 810 & - & - \\
DESN045 & 2913 & 721 & 1064 \\
DESN050 & 2939 & 710 & - \\
DESN051 & 1451 & 685 & 1075 \\
DESN057 & 1535 & - & - \\
DESN059 & 2533 & 714 & 1078 \\
DESN074 & 2534 & 702 & 1062 \\
DESN076 & 2489 & 717 & 1075 \\
DESN079 & - & - & 1071 \\
DESN085 & 713 & - & - \\
DESN092 & - & 536 & 1057 \\
DEST002 & 3020 & 721 & 1075 \\
DEST005 & 802 & - & - \\
DEST011 & 2924 & 713 & 1075 \\
DEST014 & 996 & - & - \\
DEST022 & 805 & - & - \\
DEST025 & 480 & - & - \\
DEST028 & 2694 & - & - \\
DEST030 & 2282 & - & - \\
DEST031 & 796 & - & - \\
DEST032 & 447 & - & - \\
DEST039 & 2971 & 676 & 1069 \\
DEST044 & 2923 & 709 & 1086 \\
DEST050 & 2672 & 706 & 1075 \\
DEST052 & 1484 & - & - \\
DEST061 & 814 & - & - \\
DEST063 & 1241 & - & - \\
DEST066 & 2991 & 659 & 1086 \\
DEST069 & 2589 & 707 & - \\
DEST070 & 1488 & - & - \\
DEST071 & 462 & - & - \\
DEST072 & 2641 & 703 & - \\
DEST077 & 1611 & 663 & 1086 \\
DEST078 & 3042 & 709 & - \\
DEST089 & 2467 & 710 & 1048 \\
\hline & 1422 & 649 & 1086 \\
\hline
\end{tabular}




\section{A2 Joint distributions}

Forecasts and observations are treated as random variables. Let $p(m, o)$ represent the joint distribution of a model's forecast $m$ and an observation $o$, which contains information on the forecast, the observation, and the relationship between both of them (Murphy and Winkler, 1987). To access specific pieces of information, we factorise the joint distribution into a conditional and a marginal distribution in two ways. The first factorisation is called calibration refinement and reads

$p(m, o)=p(o \mid m) p(m)$,

where $p(o \mid m)$ is the conditional distribution of observing $o$ given the forecast $m$, and $p(m)$ is the marginal distribution which indicates how often different forecast values are used (Murphy and Winkler, 1987; Wilks, 2006). A continuous forecast is perfectly calibrated if

$E(o \mid m)=m$

holds, where $E(o \mid m)$ is the expected value of $o$ given the forecast $m$. The marginal distribution $p(m)$ is a measure of how often different forecasts are made and is therefore also called refinement or sharpness. Both distributions are important to evaluate a model's performance. Murphy and Winkler (1987) pointed out that a perfectly calibrated forecast is worth nothing if it lacks refinement.

The second factorisation is called the likelihood-base rate and consequently is given by

$p(m, o)=p(m \mid o) p(o)$,

where $p(m \mid o)$ is the conditional distribution of forecast $m$ given that $o$ was observed. $p(o)$ is the marginal distribution which only contains information about the underlying rate of occurrence of observed values and is therefore also called the sample climatological distribution (Wilks, 2006).

\section{A3 Mean squared error decomposition (Murphy, 1988)}

This section provides additional information about the MSE decomposition introduced by Murphy (1988). The MSE decomposition is performed as

$$
\begin{aligned}
\operatorname{MSE}(\boldsymbol{m}, \boldsymbol{o}) & =\frac{1}{n} \sum_{i=1}^{n}\left(\left(m_{i}-\bar{m}\right)-\left(o_{i}-\bar{o}\right)+(\bar{m}-\bar{o})\right)^{2} \\
& =(\bar{m}-\bar{o})^{2}+\sigma_{m}^{2}+\sigma_{o}^{2}-2 \sigma_{m o} \\
& =(\bar{m}-\bar{o})^{2}+\sigma_{m}^{2}+\sigma_{o}^{2}-2 \sigma_{m} \sigma_{o} \rho_{m o}
\end{aligned}
$$

Here, $\sigma_{m}\left(\sigma_{o}\right)$ is the sample variance of the forecasts (observations) and $\sigma_{m o}$ is the sample covariance of the forecasts and observations, which is given by $\sigma_{m o}=$ $\frac{1}{n} \sum_{i=1}^{n}\left(m_{i}-\bar{m}\right)\left(o_{i}-\bar{o}\right) . \rho_{m o}$ is the sample coefficient of 
correlation between forecast and observation.

Case I:

$$
\begin{aligned}
& S(m, \bar{o}, o)= \\
& \underbrace{\rho_{m o}^{2}}_{\mathrm{AI}}-\underbrace{\left(\rho_{m o}-\frac{\sigma_{m}}{\sigma_{o}}\right)^{2}}_{\mathrm{BI}}-\underbrace{\left(\frac{\bar{m}-\bar{o}}{\sigma_{o}}\right)^{2}}_{\mathrm{CI}}
\end{aligned}
$$

Case II:

$$
\begin{aligned}
& S\left(m, o^{\star}, o\right)= \\
& \frac{\mathrm{AI}-\mathrm{BI}-\mathrm{CI}-\rho_{O^{\star} O}^{2}+\left(\rho_{O^{\star} o}-\frac{\sigma_{o^{\star}}}{\sigma_{o}}\right)^{2}}{1-\underbrace{\rho_{O^{\star} O}^{2}}_{\text {AII }}+\underbrace{\left(\rho_{O^{\star} o}-\frac{\sigma_{O^{\star}}}{\sigma_{o}}\right)^{2}}_{\text {BII }}}
\end{aligned}
$$

Case III:

$$
\begin{aligned}
& S(m, \bar{\mu}, o)= \\
& \frac{\mathrm{AI}-\mathrm{BI}-\mathrm{CI}+\left(\frac{\bar{\mu}-\bar{o}}{\sigma_{o}}\right)^{2}}{1+\underbrace{\left(\frac{\bar{\mu}-\bar{o}}{\sigma_{o}}\right)^{2}}_{\text {AIII }}} .
\end{aligned}
$$

Case IV:

$$
\begin{aligned}
& S(m, \mu, o)= \\
& \frac{\mathrm{AI}-\mathrm{BI}-\mathrm{CI}-\rho_{\mu o}^{2}+\left(\rho_{\mu o}^{2}-\frac{\sigma_{\mu}}{\sigma_{o}}\right)^{2}+\left(\frac{\bar{\mu}-\bar{o}}{\sigma_{o}}\right)^{2}}{1-\underbrace{\rho_{\mu o}^{2}}_{\text {AIV }}+\underbrace{\left(\rho_{\mu o}^{2}-\frac{\sigma_{\mu}}{\sigma_{o}}\right)^{2}}_{\text {BIV }}+\underbrace{\left(\frac{\bar{\mu}-\bar{o}}{\sigma_{o}}\right)^{2}}_{\text {CIV }}}
\end{aligned}
$$

The term AI is the square of the sample correlation coefficient and might be interpreted as the strength of linear relationship between the forecast and the observation. This term ranges from 0 (no correlation) to 1 (perfect correlation). The term BI includes the square of the differences between the sample correlation coefficient and the ratio of standard deviation of the forecast and observation. Therefore, $\mathrm{BI}$ is a measure of the conditional bias of the forecast which is always positive due to the square and tends to decrease skill as it is a subtrahend. The last term, which is included in all cases (I-IV), is CI and contains the square of the difference of the mean forecast and mean observation divided by the variance of the observation. Therefore, $\mathrm{CI}$ is a measure of the unconditional bias in the forecast and, again, tends to decrease the skill as it is a subtrahend which is always greater than or equal to zero.

In the case of multi-value internal climatology (Case II, Eq. A8), two additional terms appear in the dominator as

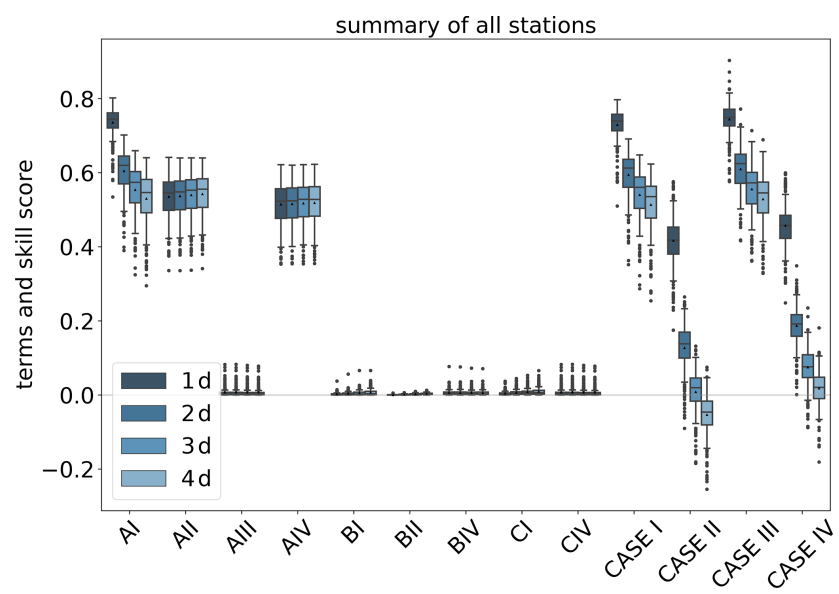

Figure A1. Skill scores of IntelliO3-ts with respect to climatological reference forecast, with internal single value reference (Case I), internal multi-value (monthly) reference (Case II), external single (Case III), and external multi-value (monthly) reference (Case IV) for all lead times from $1 \mathrm{~d}$ (dark blue) to $4 \mathrm{~d}$ (light blue). All terms are described in Sect. A3. Triangles denote the arithmetic means.

well as the numerator which tend to decrease skill in general and only vanish if $2 \rho_{o^{\star} o}=\sigma_{\mu} / \sigma_{o}$. In Case III, the additional term AIII appears that includes the square of the difference between the mean external and the internal climatology divided by the variance of the observation. AIII leads to an increase of skill for any difference in the means of external and internal climatologies.

Three additional terms (AIV, BIV, and CIV) appear if Eq. (7) is decomposed by using a multi-value external climatology as reference forecast (Case IV). These terms only vanish if $2 \rho_{\mu o}=\sigma_{\mu} / \sigma_{o}$ and $\bar{\mu}=\bar{o}$. A summary of all four cases and all terms included is given in Table A2.

Figure A1 also includes all individual terms as described above.

\section{A4 Additional information on JUWELS}

Each node on JUWELS (Jülich Supercomputing Centre, 2019) which is part of the graphical processor unit (GPU) partition is equipped with four NVIDIA Volta V100 GPUs. The user guide for JUWELS is available from https:// apps.fz-juelich.de/jsc/hps/juwels/index.html (last access: 12 November 2020).

\section{A5 Detailed model settings}

Figures A2 and A3 show the full architecture of IntelliO3ts including all individual layers and tails. Table A3 lists the specific compile options per keyword of Keras' compile method. Table A4 summarises additional settings for the specific architecture. 
Table A2. Summarised skill scores $S(\boldsymbol{m}, \boldsymbol{r}, \boldsymbol{o})$ based on the MSE (Cases I-IV) and relating terms (AI-CIV) as described in Sect. A3. $\boldsymbol{m}$ denotes the prediction model, $\boldsymbol{r}$ is the reference, and $\boldsymbol{o}$ denotes the observation. The " $x$ " sign marks if a term (AI to CIV) appears in the different factorisations. The following abbreviations are used: corr. for correlation, obs. for observation, ref. reference (forecast), cond. for conditional, int. for internal, and ext. for external.

\begin{tabular}{|c|c|c|c|c|c|c|}
\hline Term & Case I & Case II & Case III & Case IV & Formula & Meaning \\
\hline$r:$ & $\begin{array}{l}\bar{o} \\
\text { int. single }\end{array}$ & $\begin{array}{l}\boldsymbol{o}^{\star} \\
\text { int. multi }\end{array}$ & $\begin{array}{l}\bar{\mu} \\
\text { ext. single }\end{array}$ & $\begin{array}{l}\boldsymbol{\mu} \\
\text { ext. multi }\end{array}$ & & reference \\
\hline AI & $\times$ & $\times$ & $\times$ & $\times$ & $\rho_{m o}^{2}$ & potential skill \\
\hline AII & & $x$ & & & $\rho_{O^{\star} O}^{2}$ & corr. obs.-ref. \\
\hline AIII & & & $x$ & & $\left(\frac{\bar{\mu}-\bar{o}}{\sigma_{o}}\right)^{2}$ & trend \\
\hline AIV & & & & $\times$ & $\rho_{\mu o}^{2}$ & corr. obs.-ref. \\
\hline BI & $x$ & $x$ & $x$ & $x$ & $\left(\rho_{m o}-\frac{\sigma_{m}}{\sigma_{o}}\right)^{2}$ & cond. bias \\
\hline BII & & $x$ & & & $\left(\rho_{o^{\star} O}-\frac{\sigma_{o^{\star}}}{\sigma_{o}}\right)^{2}$ & cond. bias \\
\hline BIV & & & & $\times$ & $\left(\rho_{\mu o}^{2}-\frac{\sigma_{\mu}}{\sigma_{o}}\right)^{2}$ & cond. bias \\
\hline $\mathrm{CI}$ & $\times$ & $x$ & $x$ & $x$ & $\left(\frac{\bar{m}-\bar{o}}{\sigma_{o}}\right)^{2}$ & uncond. bias \\
\hline CIV & & & & $\times$ & $\left(\frac{\bar{\mu}-\bar{o}}{\sigma_{o}}\right)^{2}$ & trend \\
\hline Equation & (A7) & (A8) & (A9) & (A10) & & \\
\hline
\end{tabular}

Table A3. Specific compile options passed to Keras' compile method. Other keywords which are not listed in this table are left with default values.

\begin{tabular}{ll}
\hline Keyword & Value \\
\hline optimizer & adam $(1 \mathrm{r}=0.001$, amsgrad=True $)$ \\
loss & Eqs. $(3),(2)$ \\
loss_weight & $0.01,0.99$
\end{tabular}

Table A4. Specific information and rates used to set up the model architecture.

\begin{tabular}{ll}
\hline Setting & Value \\
\hline Dropout rate & 0.35 \\
Regularizer & keras.regularizers.12(0.01) \\
Epochs & 300 \\
Activation (all without last layer) & ELU \\
Activation (output layers) & linear \\
Padding & symmetric padding \\
\hline
\end{tabular}

\section{A6 Seasonal decomposition of conditional quantiles}

The following section contains all conditional quantile plots decomposed for all seasons (DJF: Fig. A4a-d, MAM: Fig. A5a-d, JJA: Fig. A6a-d, and SON: Fig. A7a-d). 


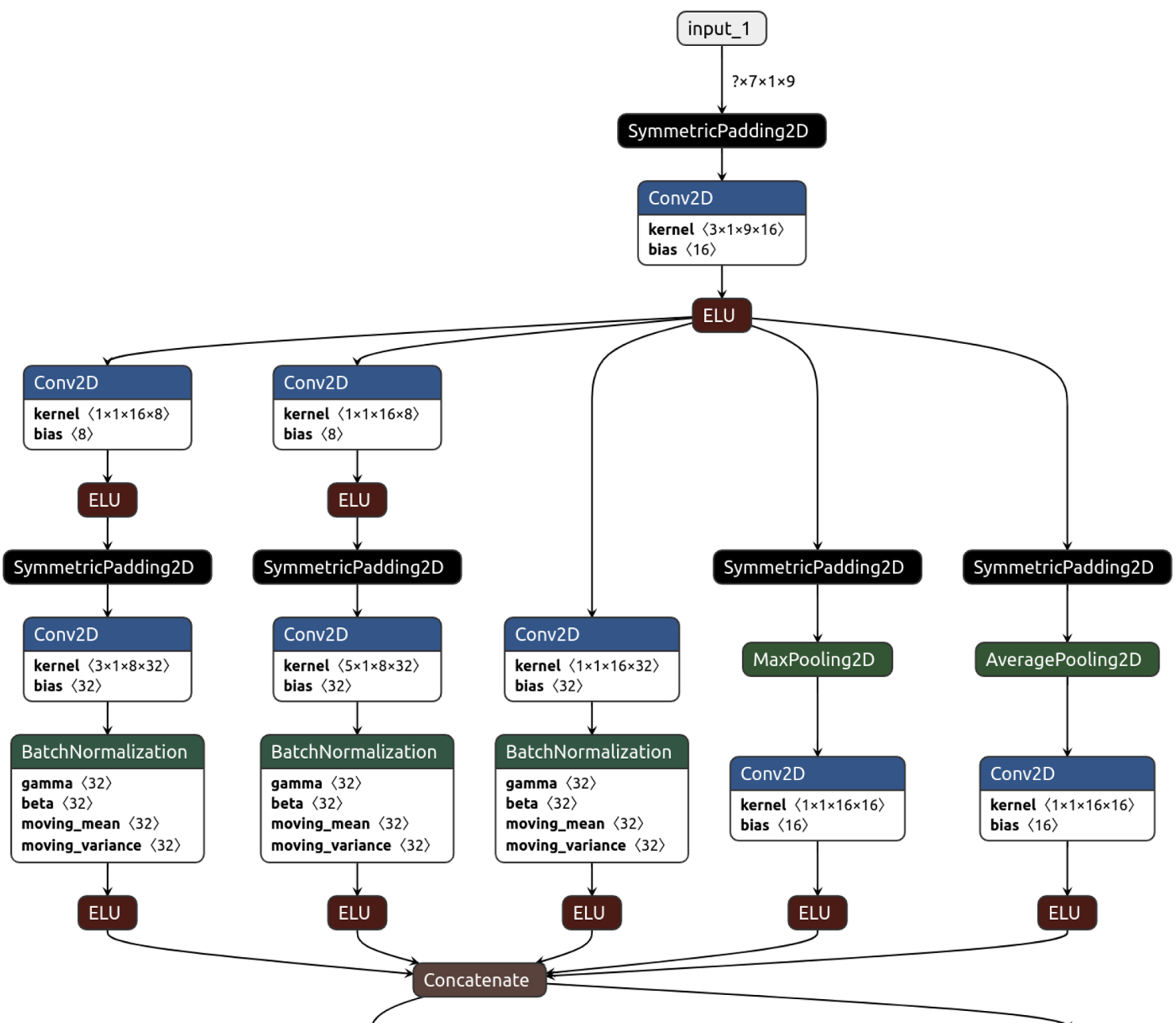

Figure A2. First part of the network showing the input, the first padding, convolution and activation, and the first inception block. This figure was created with Netron (Roeder, 2020). 


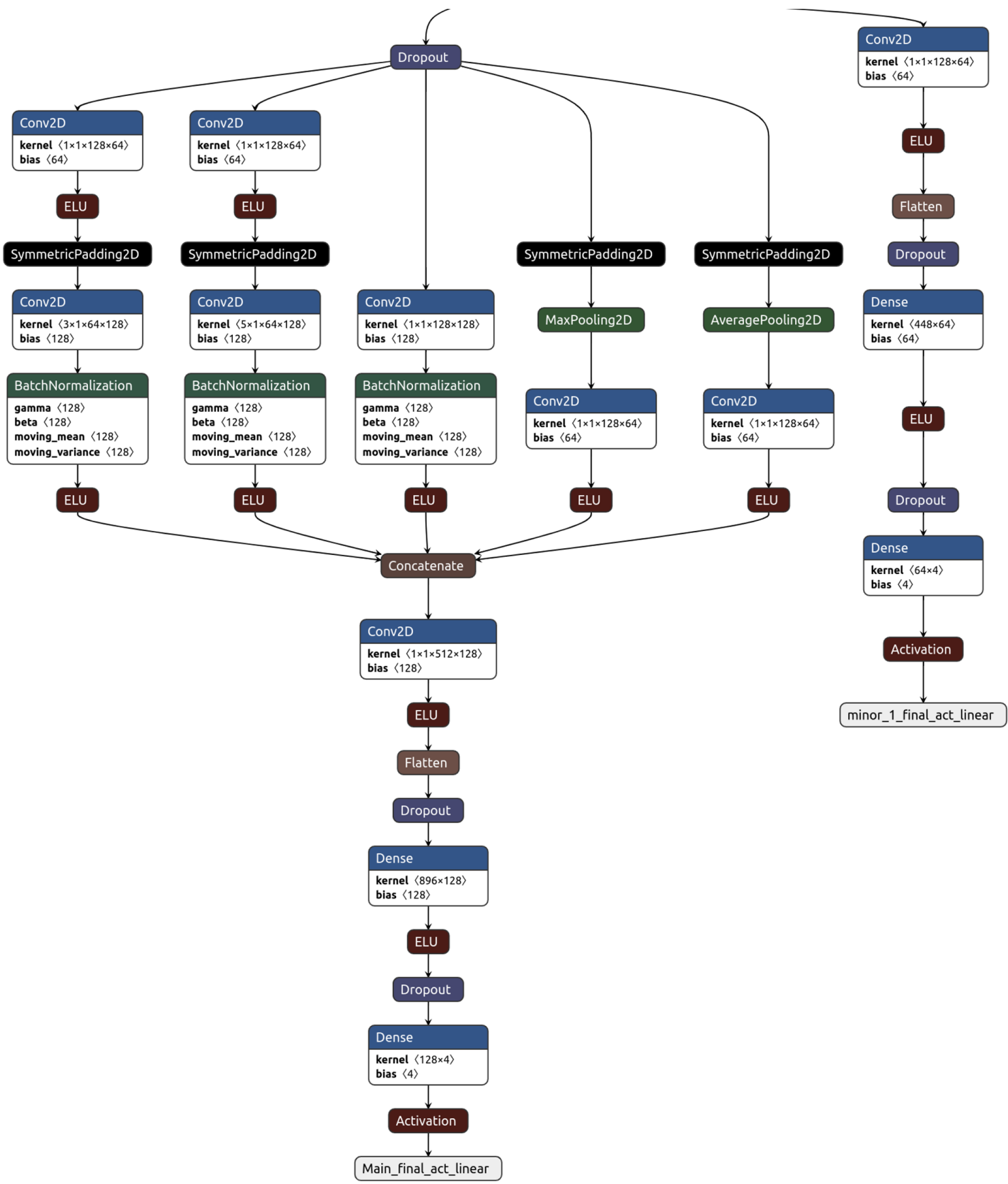

Figure A3. Second part of the network after the "concatenate" layer in Fig. A2, showing the minor output branch, the second inception block, and the main output branch. This figure was created with Netron (Roeder, 2020). 


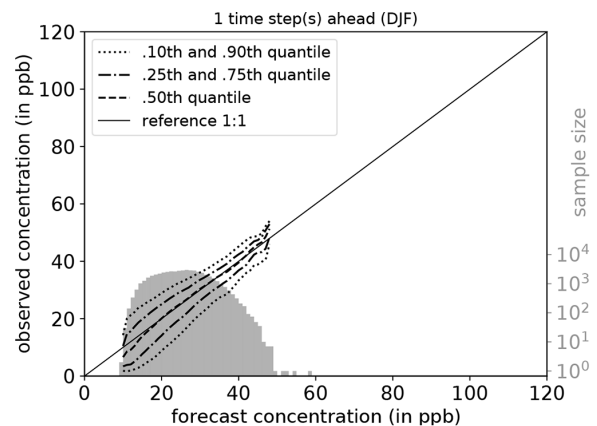

(a)

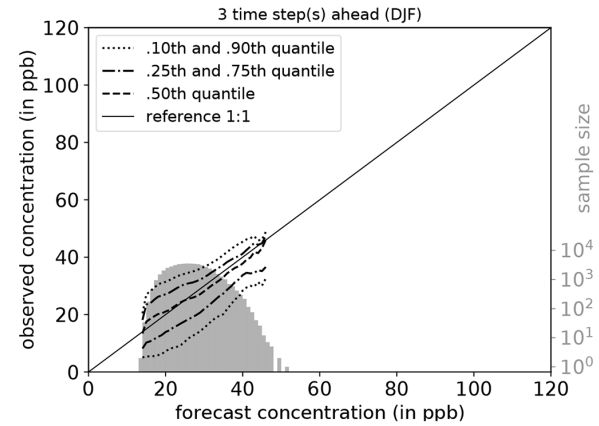

(c)

Figure A4. Same as Fig. 6 but for DJF.

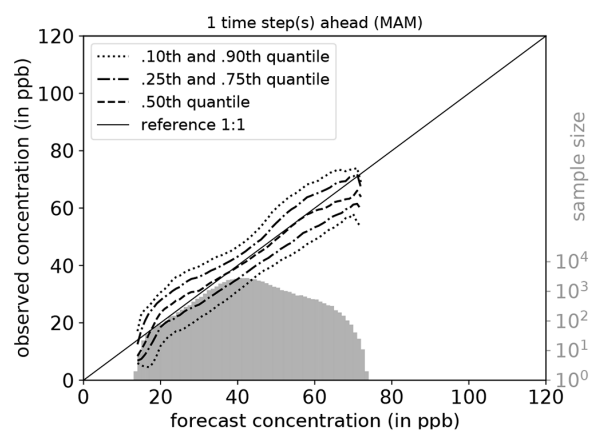

(a)

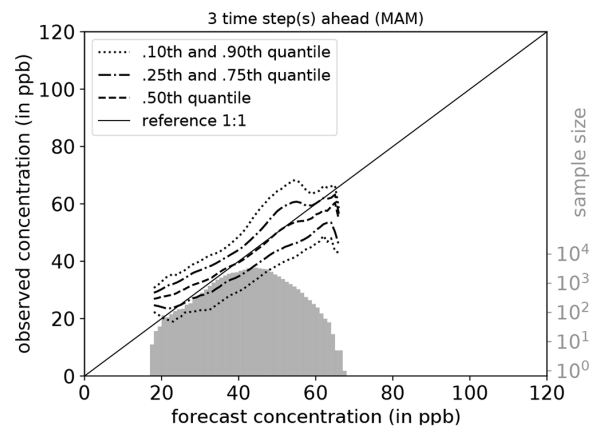

(c)

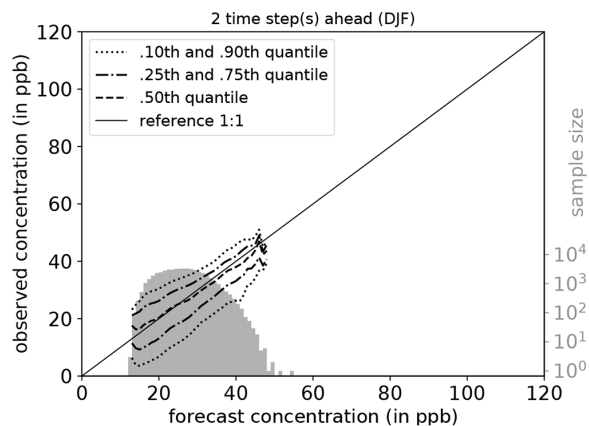

(b)

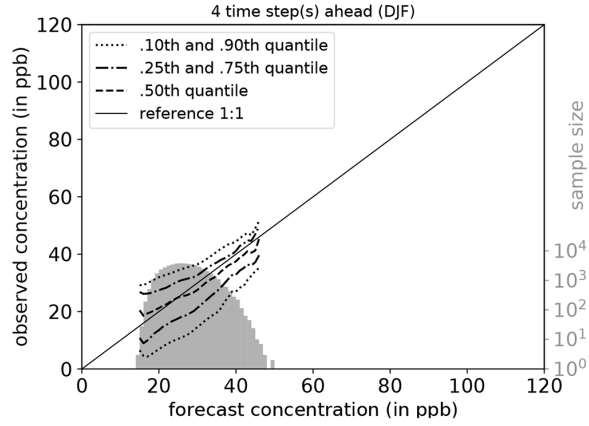

(d)

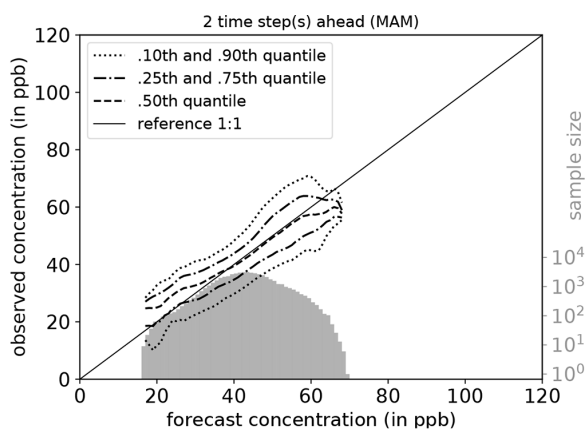

(b)

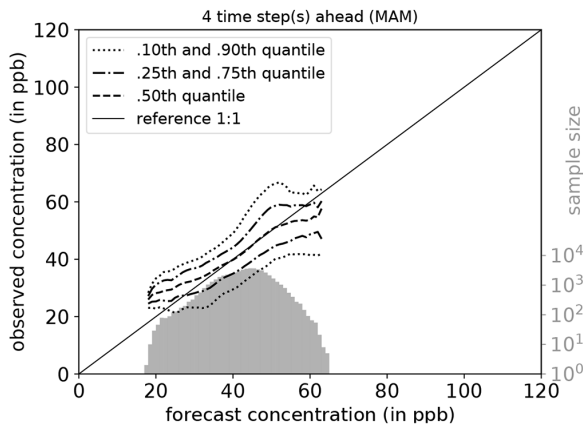

(d)

Figure A5. Same as Fig. 6 but for MAM. 


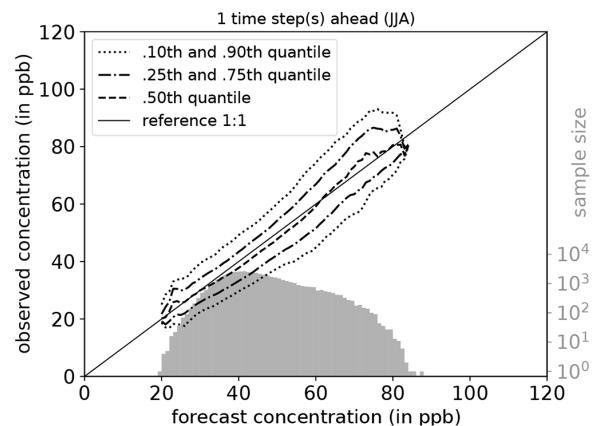

(a)

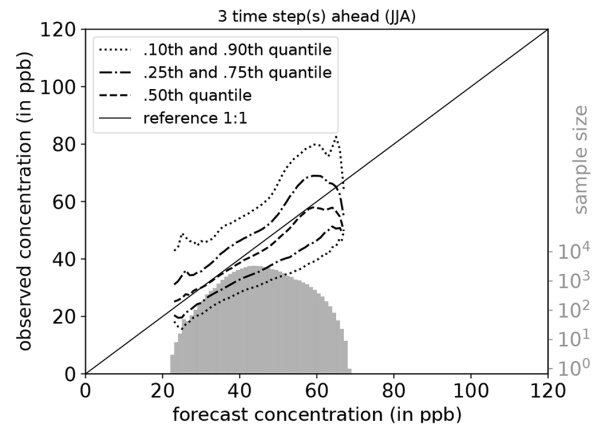

(c)

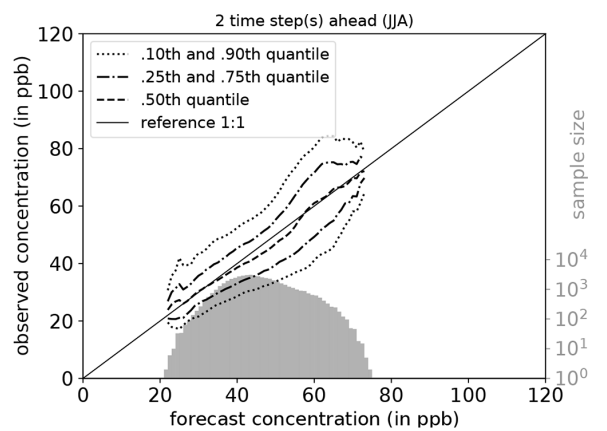

(b)

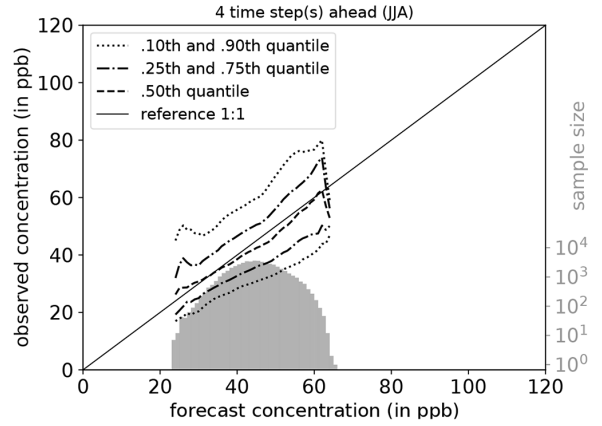

(d)

Figure A6. Same as Fig. 6 but for JJA.

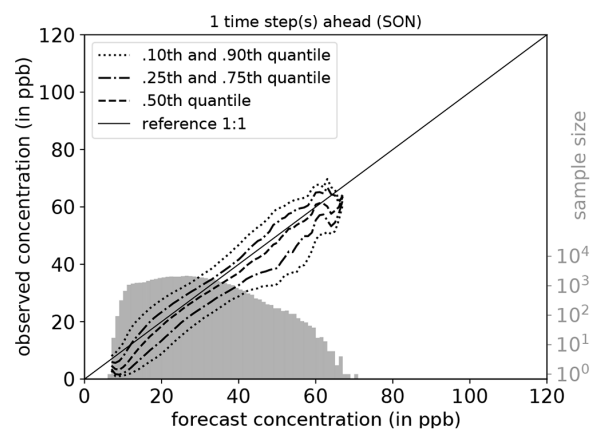

(a)

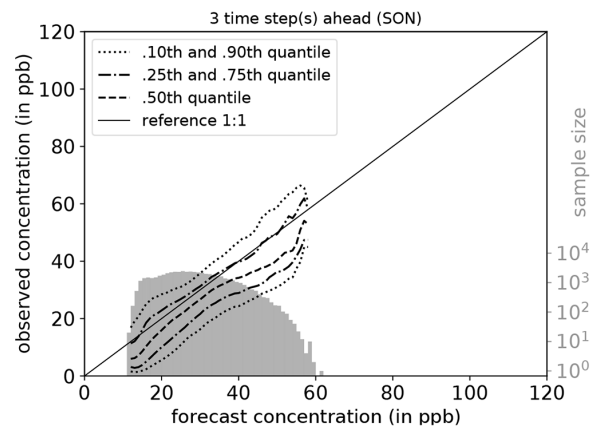

(c)

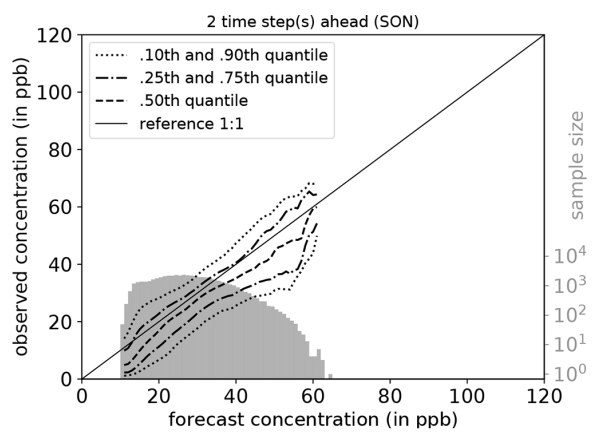

(b)

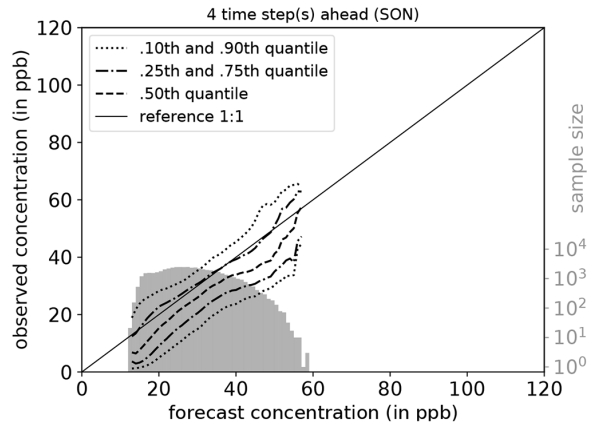

(d)

Figure A7. Same as Fig. 6 but for SON. 
Code and data availability. The current version of IntelliO3-ts is available from the project website: https://gitlab.version.fz-juelich.de/toar/mlair/-/tree/IntelliO3-ts (last access: 12 November 2020) under the MIT licence (http://opensource.org/licenses/mit-license.php, last access: 18 December 2020). The exact versions of the model and data used to produce the results in this paper are archived on b2share at https: //doi.org/10.23728/b2share.5042cda41a4c49769cc4010d231ecdec (Kleinert et al., 2020b). The initial version which was used for the initial submission is also archived on b2share at https://doi.org/10.34730/c5dae21fac954aa6bdb4e86172221526

(Kleinert et al., 2020a).

Author contributions. FK and MGS developed the concept of the study. All authors jointly developed the concept of the machine learning model. FK implemented the neural network and performed the experiment. FK had the lead in writing the manuscript with contributions from LHL and MGS. LHL had the technical lead in code development and workflow design. All authors revised the final manuscript and submitted it to GMD.

Competing interests. The authors declare that they have no conflict of interest.

Acknowledgements. We are thankful to all air quality data providers which made their data available in the TOAR database. Moreover, we thank the meteorological section of the Institute of Geosciences at the University of Bonn, which provided the COSMO reanalysis data. We thank Sabine Schröder for the help in accessing data through the JOIN interface and Jenia Jitsev for helpful discussions.

The authors gratefully acknowledge the Gauss Centre for Supercomputing e.V. (http://www.gauss-centre.eu, last access: 18 December 2020) for funding this project by providing computing time through the John von Neumann Institute for Computing (NIC) on the GCS Supercomputer JUWELS at Jülich Supercomputing Centre (JSC). We are grateful to the anonymous reviewers for the constructive review and encouraging comments.

Financial support. This research has been supported by the European Research Council (grant no. IntelliAQ (787576)).

The article processing charges for this open-access

publication were covered by a Research

Centre of the Helmholtz Association.

Review statement. This paper was edited by Juan Antonio Añel and reviewed by two anonymous referees.

\section{References}

Abadi, M., Agarwal, A., Barham, P., Brevdo, E., Chen, Z., Citro, C., Corrado, G. S., Davis, A., Dean, J., Devin, M., Ghemawat, S.,
Goodfellow, I., Harp, A., Irving, G., Isard, M., Jia, Y., Jozefowicz, R., Kaiser, M., Kudlur, M., Levenberg, J., Mané, M., Monga, R., Moore, S., Murray, D., Olah, C., Schuster, M., Shlens, J., Steiner, J., Sutskever, I., Talwar, J., Tucker, P., Vanhoucke, V., Vasudevan, V., Viégas, F., Vinyals, O., Warden, P., Wattenberg M., Wicke, M., Yu, Y., and Zheng, X.: TensorFlow: LargeScale Machine Learning on Heterogeneous Systems, available at: https://www.tensorflow.org/ (last access: 18 December 2020), 2015.

Abdul Aziz, F. A. B., Abd. Rahman, N., and Mohd Ali, J.: Tropospheric Ozone Formation Estimation in Urban City, Bangi, Using Artificial Neural Network (ANN), Comput. Intel. Neurosc., 2019, 1-10, https://doi.org/10.1155/2019/6252983, 2019.

Abdul-Wahab, S. A., Bakheit, C. S., and Al-Alawi, S. M.: Principal component and multiple regression analysis in modelling of ground-level ozone and factors affecting its concentrations, Environ. Modell. Softw., 20, 1263-1271, https://doi.org/10.1016/j.envsoft.2004.09.001, 2005.

Avnery, S., Mauzerall, D. L., Liu, J., and Horowitz, L. W.: Global crop yield reductions due to surface ozone exposure: 1. Year 2000 crop production losses and economic damage, Atmos. Environ., 45, 2284-2296, https://doi.org/10.1016/j.atmosenv.2010.11.045, 2011.

Bai, S., Kolter, J. Z., and Koltun, V.: An Empirical Evaluation of Generic Convolutional and Recurrent Networks for Sequence Modeling, arXiv [preprint], arXiv:1803.01271, 2018.

Bell, M. L., Zanobetti, A., and Dominici, F.: Who is More Affected by Ozone Pollution? A Systematic Review and Meta-Analysis, Am. J. Epidemiol., 180, 15-28, https://doi.org/10.1093/aje/kwu115, 2014.

Bollmeyer, C., Keller, J. D., Ohlwein, C., Wahl, S., Crewell, S., Friederichs, P., Hense, A., Keune, J., Kneifel, S., Pscheidt, I., Redl, S., and Steinke, S.: Towards a high-resolution regional reanalysis for the European CORDEX domain, Q. J. Roy. Meteor. Soc., 141, 1-15, https://doi.org/10.1002/qj.2486, 2015.

Brunner, D., Savage, N., Jorba, O., Eder, B., Giordano, L., Badia, A., Balzarini, A., Baró, R., Bianconi, R., Chemel, C., Curci, G., Forkel, R., Jiménez-Guerrero, P., Hirtl, M., Hodzic, A., Honzak, L., Im, U., Knote, C., Makar, P., Manders-Groot, A., van Meijgaard, E., Neal, L., Pérez, J. L., Pirovano, G., San Jose, R., Schröder, W., Sokhi, R. S., Syrakov, D., Torian, A., Tuccella, P., Werhahn, J., Wolke, R., Yahya, K., Zabkar, R., Zhang, Y., Hogrefe, C., and Galmarini, S.: Comparative analysis of meteorological performance of coupled chemistry-meteorology models in the context of AQMEII phase 2, Atmos. Environ., 115, 470-498, https://doi.org/10.1016/j.atmosenv.2014.12.032, 2015.

Chollet, F.: Keras, available at: https://keras.io (last access: 18 December 2020), 2015.

Clevert, D.-A., Unterthiner, T., and Hochreiter, S.: Fast and Accurate Deep Network Learning by Exponential Linear Units (ELUs), arXiv [preprint], arXiv:1511.07289, 2016.

Cobourn, W. G., Dolcine, L., French, M., and Hubbard, M. C.: A Comparison of Nonlinear Regression and Neural Network Models for Ground-Level Ozone Forecasting, J. Air Waste Ma., 50, 1999-2009, https://doi.org/10.1080/10473289.2000.10464228, 2000.

Collins, W. J., Stevenson, D. S., Johnson, C. E., and Derwent, R. G.: Tropospheric Ozone in a Global-Scale Three-Dimensional Lagrangian Model and Its Response to 
NOX Emission Controls, J. Atmos. Chem., 26, 223-274, https://doi.org/10.1023/A:1005836531979, 1997.

Comrie, A. C.: Comparing Neural Networks and Regression Models for Ozone Forecasting, J. Air Waste Ma., 47, 653-663, https://doi.org/10.1080/10473289.1997.10463925, 1997.

Dauphin, Y. N., Fan, A., Auli, M., and Grangier, D.: Language Modeling with Gated Convolutional Networks, arXiv [preprint], arXiv:1612.08083, 2017.

Donner, L. J., Wyman, B. L., Hemler, R. S., Horowitz, L. W., Ming, Y., Zhao, M., Golaz, J.-C., Ginoux, P., Lin, S.-J., Schwarzkopf, M. D., Austin, J., Alaka, G., Cooke, W. F., Delworth, T. L., Freidenreich, S. M., Gordon, C. T., Griffies, S. M., Held, I. M., Hurlin, W. J., Klein, S. A., Knutson, T. R., Langenhorst, A. R., Lee, H.-C., Lin, Y., Magi, B. I., Malyshev, S. L., Milly, P. C. D., Naik, V., Nath, M. J., Pincus, R., Ploshay, J. J., Ramaswamy, V., Seman, C. J., Shevliakova, E., Sirutis, J. J., Stern, W. F., Stouffer, R. J., Wilson, R. J., Winton, M., Wittenberg, A. T., and Zeng, F.: The Dynamical Core, Physical Parameterizations, and Basic Simulation Characteristics of the Atmospheric Component AM3 of the GFDL Global Coupled Model CM3, J. Climate, 24, 34843519, https://doi.org/10.1175/2011JCLI3955.1, 2011.

Dye, T. S.: Guidelines for developing an air quality (ozone and $\mathrm{PM}_{2.5}$ ) forecasting program, US Environmental Protection Agency, Office of Air Quality Planning and Standards, Information Transfer and Program Integration Division, AIRNow Program, available at: https://nepis.epa.gov/Exe/ZyPURL.cgi? Dockey=2000F0ZT.txt (last access: 18 December 2020), 2003.

Eslami, E., Choi, Y., Lops, Y., and Sayeed, A.: A realtime hourly ozone prediction system using deep convolutional neural network, Neural Comput. Appl., 32, 8783-8797, https://doi.org/10.1007/s00521-019-04282-x, 2020.

Fleming, Z. L., Doherty, R. M., Von Schneidemesser, E., Malley, C. S., Cooper, O. R., Pinto, J. P., Colette, A., Xu, X., Simpson, D., Schultz, M. G., Lefohn, A. S., Hamad, S., Moolla, R., Solberg, S., and Feng, Z.: Tropospheric Ozone Assessment Report: Present-day ozone distribution and trends relevant to human health, Elem. Sci. Anth., 6, 12, https://doi.org/10.1525/elementa.273, 2018.

Fuentes, M. and Raftery, A. E.: Model Evaluation and Spatial Interpolation by Bayesian Combination of Observations with Outputs from Numerical Models, Biometrics, 61, 36-45, https://doi.org/10.1111/j.0006-341X.2005.030821.x, 2005.

Gardner, M. and Dorling, S.: Artificial Neural NetworkDerived Trends in Daily Maximum Surface Ozone Concentrations, J. Air Waste Ma., 51, 1202-1210, https://doi.org/10.1080/10473289.2001.10464338, 2001.

Grell, G. A., Peckham, S. E., Schmitz, R., McKeen, S. A., Frost, G., Skamarock, W. C., and Eder, B.: Fully coupled "online" chemistry within the WRF model, Atmos. Environ., 39, 6957-6975, https://doi.org/10.1016/j.atmosenv.2005.04.027, 2005.

Horowitz, J. and Barakat, S.: Statistical analysis of the maximum concentration of an air pollutant: Effects of autocorrelation and non-stationarity, Atmos. Environ., 13, 811-818, https://doi.org/10.1016/0004-6981(79)90272-5, 1979.

Horowitz, L. W., Stacy, W., Mauzerall, D. L., Emmons, L. K., Rasch, P. J., Granier, C., Tie, X., Lamarque, J., Schultz, M. G., Tyndall, G. S., Orlando, J. J., and Brasseur, G. P.: A global simulation of tropospheric ozone and related tracers: Description and evaluation of MOZART, version 2, J. Geophys. Res.-Atmos., 108, D12, https://doi.org/10.1029/2002JD002853, 2003.

Ioffe, S. and Szegedy, C.: Batch Normalization: Accelerating Deep Network Training by Reducing Internal Covariate Shift, arXiv [preprint], arXiv:1502.03167, 2015.

Ismail Fawaz, H., Forestier, G., Weber, J., Idoumghar, L., and Muller, P.-A.: Deep learning for time series classification: a review, Data Min. Knowl. Disc., 33, 917-963, https://doi.org/10.1007/s10618-019-00619-1, 2019.

Jacobson, M. Z.: Fundamentals of Atmospheric Modeling, Cambridge University Press, Cambridge, UK, 2005.

Jülich Supercomputing Centre: JUWELS: Modular Tier-0/1 Supercomputer at Jülich Supercomputing Centre, Journal of largescale research facilities, 5, A135, https://doi.org/10.17815/jlsrf5-171, 2019.

Kingma, D. P. and Ba, J.: Adam: A Method for Stochastic Optimization, arXiv [preprint], arXiv:1412.6980, 2014.

Kleinert, F., Leufen, L. H., and Schultz, M. G.: IntelliO3-ts: Data, b2share, https://doi.org/10.34730/c5dae21fac954aa6bdb4e86172221526, 2020a.

Kleinert, F., Leufen, L. H., and Schultz, M. G.: IntelliO3-ts: Source code and data, https://doi.org/10.23728/b2share.5042cda41a4c49769cc4010d2 31 ecdec, b2share, 2020b.

LeCun, Y., Bottou, L., Bengio, Y., and Haffner, P.: Gradient-based learning applied to document recognition, in: Proceedings of the IEEE, 86, 2278-2324, https://doi.org/10.1109/5.726791, 1998.

Lefohn, A. S., Malley, C. S., Simon, H., Wells, B., Xu, X., Zhang, L., and Wang, T.: Responses of human health and vegetation exposure metrics to changes in ozone concentration distributions in the European Union, United States, and China, Atmos. Environ., 152, 123-145, https://doi.org/10.1016/j.atmosenv.2016.12.025, 2017.

Liu, B., Yan, S., Li, J., Qu, G., Li, Y., Lang, J., and Gu, R.: A Sequence-to-Sequence Air Quality Predictor Based on the n-Step Recurrent Prediction, IEEE Access, 7, 43331-43345, https://doi.org/10.1109/ACCESS.2019.2908081, 2019.

Ma, J., Li, Z., Cheng, J. C., Ding, Y., Lin, C., and Xu, Z.: Air quality prediction at new stations using spatially transferred bidirectional long short-term memory network, Sci. Total Environ., 705, 135771, https://doi.org/10.1016/j.scitotenv.2019.135771, 2020.

Maleki, H., Sorooshian, A., Goudarzi, G., Baboli, Z., Tahmasebi Birgani, Y., and Rahmati, M.: Air pollution prediction by using an artificial neural network model, Clean Technol. Envir., 21, 1341-1352, https://doi.org/10.1007/s10098-019-01709w, 2019.

Met Office: Cartopy: a cartographic python library with a Matplotlib interface, Exeter, Devon, available at: https://scitools.org. uk/cartopy (last access: 18 December 2020), 2010-2015.

Mills, G., Sharps, K., Simpson, D., Pleijel, H., Broberg, M., Uddling, J., Jaramillo, F., Davies, W. J., Dentener, F., Van den Berg, M., Agrawal, M., Agrawal, S., Ainsworth, E. A., Büker, P., Emberson, L., Feng, Z., Harmens, H., Hayes, F., Kobayashi, K., Paoletti, E., and Van Dingenen, R.: Ozone pollution will compromise efforts to increase global wheat production, Glob. Change Biol., 24, 3560-3574, https://doi.org/10.1111/gcb.14157, 2018. 
Murphy, A. H.: Skill Scores Based on the Mean Square Error and Their Relationships to the Correlation Coefficient, Mon. Weather Rev., 116, 2417-2424, https://doi.org/10.1175/15200493(1988)116<2417:SSBOTM>2.0.CO;2, 1988.

Murphy, A. H. and Winkler, R. L.: A General Framework for Forecast Verification, Mon. Weather Rev., 115, 1330-1338, https://doi.org/10.1175/15200493(1987)115<1330:AGFFFV>2.0.CO;2, 1987.

Murphy, A. H., Brown, B. G., and Chen, Y.-S.: Diagnostic Verification of Temperature Forecasts, Weather Forecast., 4, 485-501, https://doi.org/10.1175/15200434(1989)004<0485:DVOTF>2.0.CO;2, 1989.

Olszyna, K., Luria, M., and Meagher, J.: The correlation of temperature and rural ozone levels in southeastern USA, Atmos. Environ., 31, 3011-3022, https://doi.org/10.1016/S13522310(97)00097-6, 1997.

Pawlak, I. and Jarosławski, J.: Forecasting of Surface Ozone Concentration by Using Artificial Neural Networks in $\mathrm{Ru}-$ ral and Urban Areas in Central Poland, Atmosphere, 10, 52, https://doi.org/10.3390/atmos10020052, 2019.

Prybutok, V. R., Yi, J., and Mitchell, D.: Comparison of neural network models with ARIMA and regression models for prediction of Houston's daily maximum ozone concentrations, Eur. J. Oper. Res., 122, 31-40, https://doi.org/10.1016/S03772217(99)00069-7, 2000.

Rasp, S. and Lerch, S.: Neural Networks for Postprocessing Ensemble Weather Forecasts, Mon. Weather Rev., 146, 3885-3900, https://doi.org/10.1175/MWR-D-18-0187.1, 2018.

Roeder, L.: Netron, github, available at: https:/github.com/ lutzroeder/netron, last access: 18 December 2020.

Sayeed, A., Choi, Y., Eslami, E., Lops, Y., Roy, A., and Jung, J.: Using a deep convolutional neural network to predict 2017 ozone concentrations, 24 hours in advance, Neural Networks, 121, 396408, https://doi.org/10.1016/j.neunet.2019.09.033, 2020.

Schmidhuber, J.: Deep learning in neural networks: An overview, Neural Networks, 61, 85-117, https://doi.org/10.1016/j.neunet.2014.09.003, 2015.

Schultz, M. G., Schröder, S., Lyapina, O., Cooper, O., Galbally, I., Petropavlovskikh, I., Von Schneidemesser, E., Tanimoto, H., Elshorbany, Y., Naja, M., Seguel, R., Dauert, U., Eckhardt, P., Feigenspahn, S., Fiebig, M., Hjellbrekke, A.-G., Hong, Y.-D., Christian Kjeld, P., Koide, H., Lear, G., Tarasick, D., Ueno, M., Wallasch, M., Baumgardner, D., Chuang, M.-T., Gillett, R., Lee, M., Molloy, S., Moolla, R., Wang, T., Sharps, K., Adame, J. A., Ancellet, G., Apadula, F., Artaxo, P., Barlasina, M., Bogucka, M., Bonasoni, P., Chang, L., Colomb, A., Cuevas, E., Cupeiro, M., Degorska, A., Ding, A., Fröhlich, M., Frolova, M., Gadhavi, H., Gheusi, F., Gilge, S., Gonzalez, M. Y., Gros, V., Hamad, S. H., Helmig, D., Henriques, D., Hermansen, O., Holla, R., Huber, J., Im, U., Jaffe, D. A., Komala, N., Kubistin, D., Lam, K.-S., Laurila, T., Lee, H., Levy, I., Mazzoleni, C., Mazzoleni, L., McClure-Begley, A., Mohamad, M., Murovic, M., NavarroComas, M., Nicodim, F., Parrish, D., Read, K. A., Reid, N., Ries, L., Saxena, P., Schwab, J. J., Scorgie, Y., Senik, I., Simmonds, P., Sinha, V., Skorokhod, A., Spain, G., Spangl, W., Spoor, R., Springston, S. R., Steer, K., Steinbacher, M., Suharguniyawan, E., Torre, P., Trickl, T., Weili, L., Weller, R., Xu, X., Xue, L., and Zhiqiang, M.: Tropospheric Ozone Assessment Report: Database and Metrics Data of Global Surface Ozone Observations, Elementa, 5, 58, https://doi.org/10.1525/elementa.244, 2017.

Seabold, S. and Perktold, J.: Statsmodels: Econometric and statistical modeling with python, in: Proceedings of the 9th Python in Science Conference, 28 June-3 July, Austin, Texas, 92-96, https://doi.org/10.25080/Majora-92bf1922-011, 2010.

Seinfeld, J. H. and Pandis, S. N.: Atmospheric Chemistry and Physics: From Air Pollution to Climate Change, Wiley, Hoboken, New Jersey, USA, 2016.

Seltzer, K. M., Shindell, D. T., Kasibhatla, P., and Malley, C. S.: Magnitude, trends, and impacts of ambient long-term ozone exposure in the United States from 2000 to 2015, Atmos. Chem. Phys., 20, 1757-1775, https://doi.org/10.5194/acp-201757-2020, 2020.

Silva, S. J., Heald, C. L., Ravela, S., Mammarella, I., and Munger, J. W.: A Deep Learning Parameterization for Ozone Dry Deposition Velocities, Geophys. Res. Lett., 46, 983-989, https://doi.org/10.1029/2018GL081049, 2019.

Srivastava, N., Hinton, G., Krizhevsky, A., Sutskever, I., and Salakhutdinov, R.: Dropout: a simple way to prevent neural networks from overfitting, J. Mach. Learn. Res., 15, 1929-1958, 2014.

Szegedy, C., Liu, W., Jia, Y., Sermanet, P., Reed, S., Anguelov, D., Erhan, D., Vanhoucke, V., and Rabinovich, A.: Going deeper with convolutions, in: Proceedings of the 2015 IEEE Conference on Computer Vision and Pattern Recognition (CVPR), Boston, MA, USA, 7-12 June 2015, 1-9, https://doi.org/10.1109/CVPR.2015.7298594, 2015.

Thompson, M. L., Reynolds, J., Cox, L. H., Guttorp, P., and Sampson, P. D.: A review of statistical methods for the meteorological adjustment of tropospheric ozone, Atmos. Environ., 35, 617630, https://doi.org/10.1016/S1352-2310(00)00261-2, 2001.

US Environmental Protection Agency: Technical Assistance Document for the Reporting of Daily Air Quality - the Air Quality Index (AQI), available at: https://www.airnow.gov/sites/default/ files/2018-05/aqi-technical-assistance-document-may2016.pdf (last access: 18 December 2020), 2016.

Vautard, R.: Evaluation of the meteorological forcing used for the Air Quality Model Evaluation International Initiative (AQMEII) air quality simulations, Atmos. Environ., 53, 15-37, https://doi.org/10.1016/j.atmosenv.2011.10.065, 2012.

von Kuhlmann, R., Lawrence, M. G., Crutzen, P. J., and Rasch, P. J.: A model for studies of tropospheric ozone and nonmethane hydrocarbons: Model description and ozone results, J. Geophys. Res.-Atmos., 108, D9, https://doi.org/10.1029/2002JD002893, 2003.

Wang, Y., Jacob, D. J., and Logan, J. A.: Global simulation of tropospheric $\mathrm{O}_{3}-\mathrm{NO}_{x}$-hydrocarbon chemistry: 1. Model formulation, J. Geophys. Res.-Atmospheres, 103, 10713-10725, https://doi.org/10.1029/98JD00158, 1998a.

Wang, Y., Logan, J. A., and Jacob, D. J.: Global simulation of tropospheric $\mathrm{O}_{3}-\mathrm{NO}_{x}$-hydrocarbon chemistry: 2. Model evaluation and global ozone budget, J. Geophys. Res.-Atmos., 103, 1072710755, https://doi.org/10.1029/98JD00157, 1998b.

WHO: Health risks of air pollution in Europe - HRAPIE project, Recommendations for concentration-response functions for cost-benefit analysis of particulate matter, ozone and nitrogen dioxide, Technical Report, WHO Regional Office for Europe, Copenhagen, Denmark, available at: 
http://www.euro.who.int/_data/assets/pdf_file/0006/238956/ Health_risks_air_pollution_HRAPIE_project.pdf?ua=1, (last access: 18 December 2020), 2013.

Wilks, D. S.: Statistical methods in the atmospheric sciences, International Geophysics Series, Elsevier, USA, UK, ISBN 978-0-12751966-1, 2006.

Yan, Y., Pozzer, A., Ojha, N., Lin, J., and Lelieveld, J.: Analysis of European ozone trends in the period 1995-2014, Atmos. Chem. Phys., 18, 5589-5605, https://doi.org/10.5194/acp18-5589-2018, 2018.
Zhang, Q., Lam, J. C., Li, V. O., and Han, Y.: Deep-AIR: A Hybrid CNN-LSTM Framework for Fine-Grained Air Pollution Forecast, arXiv [preprint], arXiv:2001.11957, 2020. 